\title{
Gradient Calculations for Dynamic Recurrent Neural Networks: A Survey
}

\author{
Barak A. Pearlmutter
}

\begin{abstract}
We survey learning algorithms for recurrent neural networks with hidden units, and put the various techniques into a common framework. We discuss fixedpoint learning algorithms, namely recurrent backpropagation and deterministic Boltzmann Machines, and non-fixedpoint algorithms, namely backpropagation through time, Elman's history cutoff, and Jordan's output feedback architecture. Forward propagation, an online technique that uses adjoint equations, and variations thereof, are also discussed. In many cases, the unified presentation leads to generalizations of various sorts. We discuss advantages and disadvantages of temporally continuous neural networks in contrast to clocked ones, continue with some "tricks of the trade" for training, using, and simulating continuous time and recurrent neural networks. We present some simulations, and at the end, address issues of computational complexity and learning speed.
\end{abstract}

Keywords - Recurrent neural networks, backpropagation through time, real time recurrent learning, trajectory learning.

\section{INTRODUCTION}

\section{A. Why Recurrent Networks}

The motivation for exploring recurrent architectures is their potential for dealing with two sorts of temporal behavior. First, recurrent networks are capable of settling to a solution that satisfies many constraints [1], as in a vision system which relaxes to an interpretation of an image which maximally satisfies a complex set of conflicting constraints [2], [3], [4], [5], [6], a system which relaxes to find a posture for a robot satisfying many criteria [7], and models of language parsing [8]. Although algorithms suitable for building systems of this type are reviewed to some extent below, such as the algorithm used in [9], the bulk of this paper is concerned with the problem of causing networks to exhibit particular desired detailed temporal behavior, which has found application in signal processing [10], [11], speech and language processing [12], [13], [14], and neuroscience [15], [16], [17].

It should be noted by engineers that many real-world problems which one might think would require recurrent architectures for their solution turn out to be solvable with feedforward architectures, sometimes augmented with preprocessed inputs such as tapped delay lines, and various other architectural embellishments [18], [19], [20], [21], [22], [23], [24], [25], [26], [27], [28], [29], [30], [31], [10], [32], [33], [34], [35]. For this reason, if one is interested in solving a particular problem, it would be only prudent to try a variety of non-recurrent architectures before resorting to the more powerful and general recurrent networks.

B. Pearlmutter is with the Learning Systems Department at Siemens Corporate Research, 755 College Road East, Princeton, NJ 08540. E-mail: bap@learning.siemens.com
This paper is concerned with learning algorithms for recurrent networks themselves, and not with recurrent networks as elements of larger systems, such as specialized architectures for control [36], [37], [38], [39]. Also, since we are concerned with learning, we will not discuss the computational power of recurrent networks considered as abstract machines [40], [41], [42]. Although we consider techniques for trajectory learning, we will not review practical applications thereof. In particular, grammar learning, although intriguing and progressing rapidly [43], [44], [45], [46], [47], [48], [49], typically involves recurrent neural networks as components of more complex systems, and also at present is inferior in practice to discrete algorithmic techniques [50], [51]. Grammar learning is therefore beyond our scope here. Similarly, learning of multiscale phenomena, which again typically consists of larger systems containing recurrent networks as components [52], [53], [54], [55], will not be discussed.

\section{B. Why Hidden Units}

We will restrict our attention to training procedures for networks which may include hidden units, units which have no particular desired behavior and are not directly involved in the input or output of the network. For the biologically inclined, they can be thought of as interneurons.

With the practical successes of backpropagation, it seems gratuitous to expound the virtues of hidden units and internal representations. Hidden units make it possible for networks to discover and exploit regularities of the task at hand, such as symmetries or replicated structure [56], [57], and training procedures capable of exploiting hidden units, such as the Boltzmann machine learning procedure [58] and backpropagation [59], [60], [61], [62], are behind much of the current excitement in the neural network field [63]. Also, training algorithms that do not operate with hidden units, such as the Widrow-Hoff LMS procedure [64], can be used to train recurrent networks without hidden units, so recurrent networks without hidden units reduce to nonrecurrent networks without hidden units, and therefore do not need special learning algorithms.

Consider a neural network governed by the equations

$$
\frac{d y}{d t}=f(y(t), w, I(t))
$$

where $y$ is the time-varying state vector, $w$ the parameters to be modified by the learning, and $I$ a time-varying vector of external input. Given some error metric $E^{\prime}(y, t)$, our task is to modify $w$ to reduce $E=\int E^{\prime}(y, t) d t$. Our strategy will be gradient descent, so the main portion of our 
work will be finding algorithms to calculate the gradient $\nabla_{w} E$, the vector whose elements are $\partial E / \partial w_{i}$.

The above formulation is for a continuous time system. The alternative to this is a clocked system, which obeys an equation of the form $y(t+\Delta t)=f(y(t), w, I(t))$. Without loss of generality, for clocked systems we will use $\Delta t=1$, giving

$$
y(t+1)=f(y(t), w, I(t)),
$$

with $t$ an integer.

Certainly, barring high-frequency components in $I$, the behavior of (1) can be precisely duplicated by (2) with suitable choice of $f$ in the latter. For this reason, in order to determine the practical tradeoffs of one against the other, we must consider particular functional forms for $f$. We will consider the most common neural network formulation,

$$
\frac{d y_{i}}{d t}=-y_{i}+\sigma\left(x_{i}\right)+I_{i}
$$

where $y_{i}$ is the state or activation level of unit $i$,

$$
x_{i}=\sum_{j} w_{j i} y_{j}
$$

is the total input to unit $i, w_{i j}$ is the strength of the connection from unit $i$ to unit $j$, and $\sigma$ is a differentiable function. ${ }^{1}$ The initial conditions $y_{i}\left(t_{0}\right)$ and driving functions $I_{i}(t)$ are the inputs to the system.

This defines a rather general dynamic system. Even assuming that the external input terms $I_{i}(t)$ are held constant, it is possible for the system to exhibit a wide range of asymptotic behaviors. The simplest is that the system reaches a stable fixedpoint; in the next section, we will discuss two different techniques for modifying the fixedpoints of networks that exhibit them.

More complicated possible asymptotic behaviors include limit cycles and even chaos. Later, we will describe a number of gradient based training procedures that can be applied to training networks to exhibit desired limit cycles, or particular detailed temporal behavior. We will not discuss specialized non-gradient methods for learning limit cycle attractors, such as [66], [67]. Although it has been theorized that chaotic dynamics play a significant computational role in the brain [68], [69], there are no specialized training procedures for chaotic attractors in networks with hidden units. However, Crutchfield et al. [70] and Lapedes and Farber [71] have had success with the identification of chaotic systems using models without hidden state, and there is no reason to believe that learning the dynamics of chaotic systems is more difficult than learning the dynamics of non-chaotic ones.

Special learning algorithms are available for various restricted cases. There are fixedpoint learning algorithms (for details see [72], [73], [74], [75], or for a survey see [76])

\footnotetext{
${ }^{1}$ Typically $\sigma(\xi)=\left(1+e^{-\xi}\right)^{-1}$, in which case $\sigma^{\prime}(\xi)=\sigma(\xi)(1-\sigma(\xi))$, or the scaled $\sigma(\xi)=\tanh (\xi)$, in which case $\sigma^{\prime}(\xi)=(1+\sigma(\xi))(1-$ $\sigma(\xi))=1-\sigma^{2}(\xi)$. The latter symmetric squashing function is usually preferable, as it leads to a better conditioned Hessian, which speeds gradient descent [65]. However, the former was used in all the simulations presented in this paper.
}

that take advantage of the special relationships holding at a fixedpoint to reduce the storage requirements to $O(m)$, the number of weights, and the time requirements to the time required for the network to settle down. There are continuous-time feed-forward learning algorithms that are as efficient in both time and space as algorithms for pure feedforward networks, but are applicable only when $w$ is upper-triangular but not necessarily zero-diagonal, in other words, when the network is feedforward except for recurrent self-connections [77], [78], [79], [80], [25] or for a survey, [81].

Later, we will describe a number of training procedures that, for a price in space or time, do not rely on such restrictions and can be applied to training networks to exhibit desired limit cycles, or particular detailed temporal behavior.

\section{Continuous vs. Discrete Time}

We will be concerned predominantly with continuous time networks, as in (3). However, all of the learning procedures we will discuss can be equally well applied to discrete time systems, which obey equations like (2). Continuous time has advantages for expository purposes, in that the derivative of the state of a unit with respect to time is well defined, allowing calculus to be used instead of tedious explicit temporal indexing, making for simpler derivations and exposition.

When a continuous time system is simulated on a digital computer, it is usually converted into a set of simple first order difference equations, which is formally identical to a discrete time network. However, regarding the discrete time network running on the computer as a simulation of a continuous time network has a number of advantages. First, more sophisticated and faster simulation techniques than simple first order difference equations can be used [82]. Second, even if simple first order equations are used, the size of the time step can be varied to suit changing circumstances; for instance, if the network is being used for a signal processing application and faster sensors and computers become available, the size of the time step could be decreased without retraining the network. Third, because continuous time units are stiff in time, they tend to retain information better through time. Another way of putting this is that their bias in the learning theory sense is towards temporally continuous tasks, which is certainly advantageous if the task being performed is in fact temporally continuous.

Another advantage of continuous time networks is somewhat more subtle. Even for tasks which themselves have no temporal content, such as constraint satisfaction, the natural way for a recurrent network to perform the required computation is for each unit to represent nearly the same thing at nearby points in time. Using continuous time units makes this the default behavior; in the absence other forces, units will tend to retain their state through time. In contrast, in discrete time networks, there is no a priori reason for a unit's state at one point in time to have any special relationship to its state at the next point in time. 
A pleasant added benefit of units tending to maintain their states through time is that it helps make information about the past decay more slowly, speeding up learning about the relationship between temporally distant events.

\section{LEaRning in Networks with Fixedpoints}

The fixedpoint learning algorithms we will discuss assume that the networks involved converge to stable fixedpoints. ${ }^{2}$ Networks that converge to fixedpoints are interesting because of the class of things they can compute, in particular constraint satisfaction and associative memory tasks. In such tasks, the problem is usually given to the network either by the initial conditions or by a constant external input, and the answer is given by the state of the network once it has reached its fixedpoint. This is precisely analogous to the relaxation algorithms used to solve such things as steady state heat equations, except that the constraints need not have spatial structure or uniformity.

\section{A. Will a Fixedpoint Exist?}

One problem with fixedpoints is that recurrent networks do not always converge to them. However, there are a number of special cases that guarantee convergence to a fixedpoint.

- Some simple linear conditions on the weights, such as zero-diagonal symmetry $\left(w_{i j}=w_{j i}, w_{i i}=0\right)$ guarantee that the Lyopunov function

$$
L=-\sum_{i, j} w_{i j} y_{i} y_{j}+\sum_{i}\left(y_{i} \log y_{i}+\left(1-y_{i}\right) \log \left(1-y_{i}\right)\right)
$$

decreases until a fixedpoint is reached [83]. This weight symmetry condition arises naturally if weights are considered to be Bayesian constraints, as in Boltzmann Machines [84].

- A unique fixedpoint is reached regardless of initial conditions if $\sum_{i j} w_{i j}^{2}<\max \left(\sigma^{\prime}\right)$ where $\max \left(\sigma^{\prime}\right)$ is the maximal value of $\sigma^{\prime}(x)$ for any $x$ [85], but in practice much weaker bounds on the weights seem to suffice, as indicated by empirical studies of the dynamics of networks with random weights [86].

- Other empirical studies indicate that applying fixedpoint learning algorithms stabilizes networks, causing them to exhibit asymptotic fixedpoint behavior [87], [88]. There is as yet no theoretical explanation for this phenomenon, and it has not been replicated with larger networks.

One algorithm that is capable of learning fixedpoints, but does not require the network being trained to settle to a fixedpoint in order to operate, is backpropagation through time [59]. This has been used by Nowlan to train a constraint satisfaction network for the eight queens problem, where shaping was used to gradually train a discrete time

\footnotetext{
${ }^{2}$ Technically, these algorithms only require that a fixedpoint be reached, not that it be stable. However, it is unlikely (with probability zero) that a network will converge to an unstable fixedpoint, and in practice the posibility of convergence to unstable fixedpoints can be safely ignored.
}

network without hidden units to exhibit the desired attractors [89]. However, the other fixedpoint algorithms we will consider take advantage of the special properties of a fixedpoint to simplify the learning algorithm.

\section{B. Problems with Fixedpoints}

Even when it can be guaranteed that a network settles to a fixedpoint, fixedpoint learning algorithms can still run into trouble. The learning procedures discussed here all compute the derivative of some error measure with respect to the internal parameters of the network. This gradient is then used by an optimization procedure, typically some variant of gradient descent, to minimize the error. Such optimization procedures assume that the mapping from the network's internal parameters to the consequent error is continuous, and can fail spectacularly when this assumption is violated.

Consider mapping the initial conditions $\tilde{y}\left(t_{0}\right)$ to the resultant fixedpoints, $\tilde{y}\left(t_{\infty}\right)=\mathcal{F}\left(\tilde{y}\left(t_{0}\right)\right)$. Although the dynamics of the network are all continuous, $\mathcal{F}$ need not be. For purposes of visualization, consider a symmetric network, whose dynamics thus cause the state of the network to descend the energy function of equation (5). As shown schematically in figure 1 , even an infinitesimal change to the initial conditions, or to the location of a ridge, or to the slope of an intermediate point along the trajectory, can change which fixedpoint the system ends up in. In other words, $\mathcal{F}$ is not continuous. This means that as a learning algorithm changes the locations of the fixedpoints by changing the weights, it is possible for it to cross such a discontinuity, making the error jump suddenly; and this remains true no matter how gradually the weights are changed.

\section{Recurrent Backpropagation}

It was shown independently by Pineda [72] and Alemeida [73] that the error backpropagation algorithm [61], [59], [60] is a special case of a more general error gradient computation procedure. The backpropagation equations are

$$
\begin{aligned}
x_{i} & =\sum_{j} w_{j i} y_{j} \\
y_{i} & =\sigma\left(x_{i}\right)+I_{i} \\
z_{i} & =\sigma^{\prime}\left(x_{i}\right) \sum_{j} w_{i j} z_{j}+e_{i} \\
\frac{\partial E}{\partial w_{i j}} & =y_{i} z_{j}
\end{aligned}
$$

where $z_{i}$ is the ordered partial derivative of $E$ with respect to $y_{i}$ as defined in [60], $E$ is an error measure over $y\left(t_{\infty}\right)$, and $\epsilon_{i}=\partial E / \partial y_{i}\left(t_{\infty}\right)$ is the simple derivative of $E$ with respect to the final state of a unit. In the original derivations of backpropagation, the weight matrix is assumed to be triangular with zero diagonal elements, which is another way of saying that the connections are acyclic. This ensures that a fixedpoint is reached, and allows it to be computed very efficiently in a single pass through the units. But the 


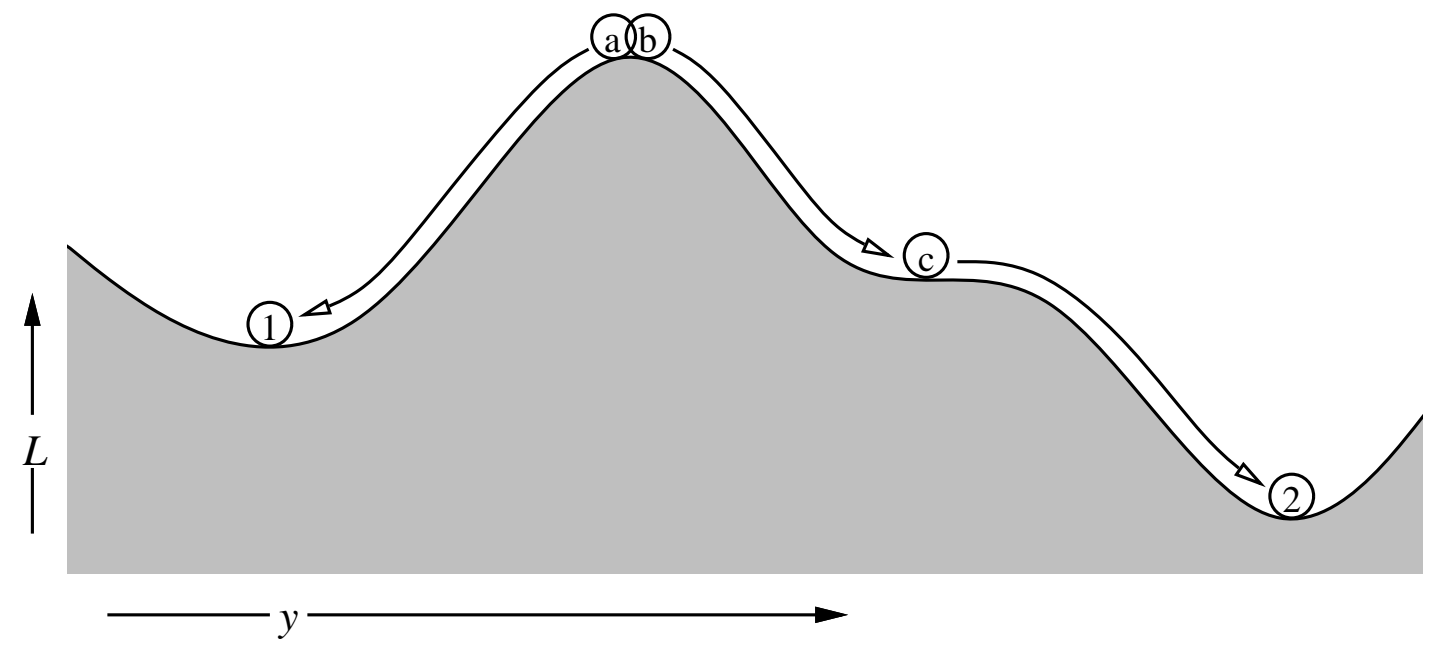

Fig. 1. This energy landscape, represented by the curved surface, and the balls, representing states of the network, illustrate some potential problems with fixedpoints. The initial conditions a and $\mathbf{b}$ can differ infinitesimally but map to different fixedpoints, so the mapping of initial conditions to fixedpoints is not continuous. Likewise, an infinitesimal change to the weights can change which fixedpoint the system evolves to from a given starting point by moving the boundary between the basins of attraction of two at tractors. Similarly, point c can be changed from a fixedpoint to a non-fixedpoint by an infinitesimal change to the weights.

backpropagation equations remain valid even with recurrent connections, assuming a fixedpoint is found.

If we assume that equation (3) reaches a fixedpoint, which we will denote $y\left(t_{\infty}\right)$, then equation (6) must be satisfied. And if (6) is satisfied, and assuming we can find $z_{i}$ that satisfy (7), then (8) will give us the derivatives we seek, even in the presence of recurrent connections. (For a simple task, [90] reports that reaching the precise fixedpoint is not crucial to learning.)

One way to compute a fixedpoint for (6) is to relax to a solution. By subtracting $y_{i}$ from each side, we get

$$
0=-y_{i}+\sigma\left(x_{i}\right)+I_{i} .
$$

At a fixedpoint, $d y_{i} / d t=0$, so the equation

$$
k \frac{d y_{i}}{d t}=-y_{i}+\sigma\left(x_{i}\right)+I_{i}
$$

has the appropriate fixedpoints. Now we note that when $-y_{i}+\sigma\left(x_{i}\right)+I_{i}$ is greater than zero, we can reduce its value by increasing $y_{i}$, so under these circumstances $d y_{i} / d t$ should be positive, so $k$ should be greater than zero. We can choose $k=1$, giving (3) as a technique for relaxing to a fixedpoint of (6).

Equation ( 7 ) is linear once $y$ is determined ( $y$ appears in the equation through the intermediate variable $x$, and also through the error terms $e_{i}$ ), so (7) has a unique solution. Any technique for solving a set of linear equations could be used. Since we are computing a fixedpoint of (6) using the associated differential equation (3), it is tempting to do the same for (7) using

$$
\frac{d z_{i}}{d t}=-z_{i}+\sigma^{\prime}\left(x_{i}\right) \sum_{j} w_{i j} z_{j}+\epsilon_{i}
$$

These equations admit to direct analog implementation. In a real analog implementation, different time constants would probably be used for (3) and (9), and under the assumption that the time $y$ and $z$ spend settling is negligible compared to the time they spend at their fixedpoints and that the rate of weight change $\eta$ is slow compared to the speed of presentation of new training samples, the weights would likely be updated continuously by an equation like

$$
\frac{d w_{i j}}{d t}=-\eta \frac{d E}{d w_{i j}}=-\eta y_{i} z_{j}
$$

or, if a momentum term $0<\alpha<1$ is desired,

$$
\frac{d^{2} w_{i j}}{d t^{2}}+(1-\alpha) \frac{d w_{i j}}{d t}+\eta y_{i} z_{j}=0 .
$$

\section{C.1 Simulation of an Associative Network}

In this section we will simulate a recurrent backpropagation network learning a higher order associative task, that of associating three pieces of information: two four bit shift registers, $A$ and $B$, and a direction bit, $D$. If $D$ is off, then $B$ is equal to $A$. If $D$ is on, then $B$ is equal to A rotated one bit to the right, with wraparound. The task is to reconstruct one of these three pieces of information, given the other two.

The architecture of the network is shown in figure 2 . Three groups of visible units hold A, B, and D. An undifferentiated group of ten hidden units is fully and bidirectionally connected to all the visible units. There are no connections between visible units. An extra unit, called a bias unit, is used to implement thresholds. This unit has no incoming connections, and is forced to always have a value of 1 by a constant external input of 0.5 . Connections 


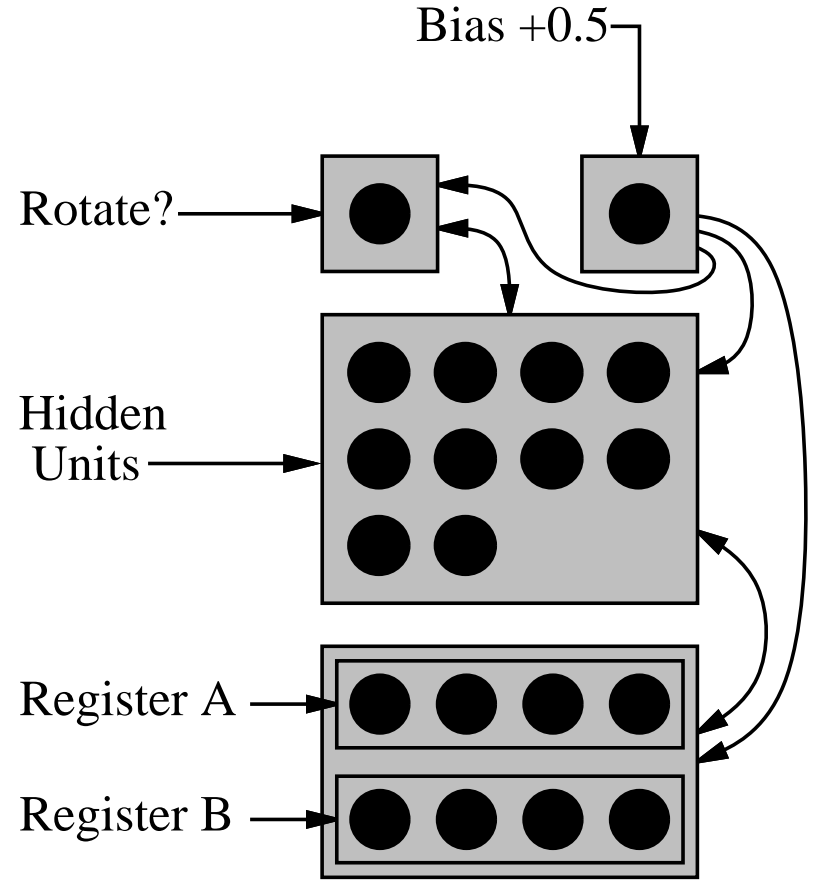

Fig. 2. The architecture of a network to solve an associative version of the four bit rotation problem.

go from it to each other unit, allowing units to have biases, which are equivalent to the negative of the threshold, without complicating the mathematics. Inputs are represented by an external input of +0.5 for an on bit, -0.5 for an off bit, and 0 for a bit to be completed by the network.

The network was trained by giving it external inputs that put randomly chosen consistent patterns on two of the three visible groups, and training the third group to attain the correct value. The error metric was the squared deviation of each $\mathrm{I} / \mathrm{O}$ unit from its desired state, except that units were not penalized for being "too correct." 3 All 96 patterns were successfully learned, except for the ones which were ambiguous, as shown in the state diagrams of figure 4 . The weights after this training, which took about 300 epochs, are shown in figure 3. By inspection, many weights are large and decidedly asymmetric; but during training, no instabilities were observed. The network consistently settled to a fixedpoint within twenty simulated time units. When the network was tested on untrained completion problems, such as reconstructing D as well as half of A and B from partially, but unambiguously, specified $\mathrm{A}$ and $\mathrm{B}$, performance was poor. However, redoing the training with weight symmetry enforced, however, caused the network to learn not only the training data but also to do well on these untrained completions.

[9] successfully applied the [72], [73] recurrent backprop-

\footnotetext{
${ }^{3}$ A unit with external input could be pushed outside the $[0,1]$
} bounds of the range of the $\sigma(\cdot)$ used.

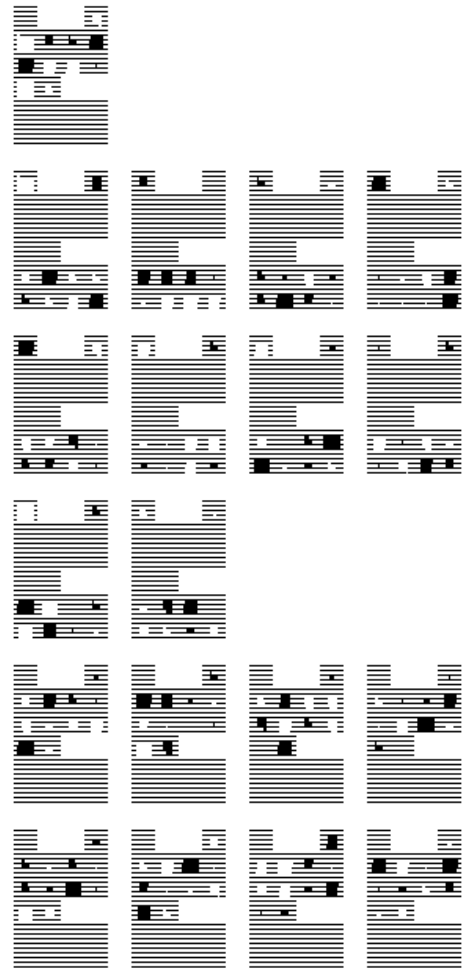

Fig. 3. A Hinton diagram of weights learned by the network of figure 2 .

agation learning procedure to learning weights for a relaxation procedure for dense stereo disparity problems with transparent surfaces. By training on examples, they were able to learn appropriate weights instead of deriving them from a simplified and unrealistic analytical model of the distribution of surfaces to be encountered, as is usual.

\section{Deterministic Boltzmann Machines}

The Mean Field form of the stochastic Boltzmann Machine learning rule, or MFT Boltzmann Machines, [91] have been shown to descend an error functional [74]. Stochastic Boltzmann Machines themselves [58] are beyond our scope here; instead, we give only the probabilistic interpretation of MFT Boltzmann Machines, without derivation.

In a a deterministic Boltzmann Machine, the transfer function of $(3)$ is $\sigma(\xi)=\left(1+e^{-\xi / T}\right)^{-1}$, where $T$ is the temperature, which starts at a high value and is gradually lowered to a target temperature each time the network is presented with a new input; without loss of generality, we assume this target temperature to be $T=1$. The weights are assumed to be symmetric and zero-diagonal. Input is handled in a different way than in the other procedures we discuss: the external inputs $I_{i}$ are set to zero, and a subset of the units, rather than obeying (3), have their values set externally. Such units are said to be clamped.

In learning, a set of input units (states over which we will index with $\alpha$ ) are clamped to some values, the output units are similarly clamped to their correct corresponding 

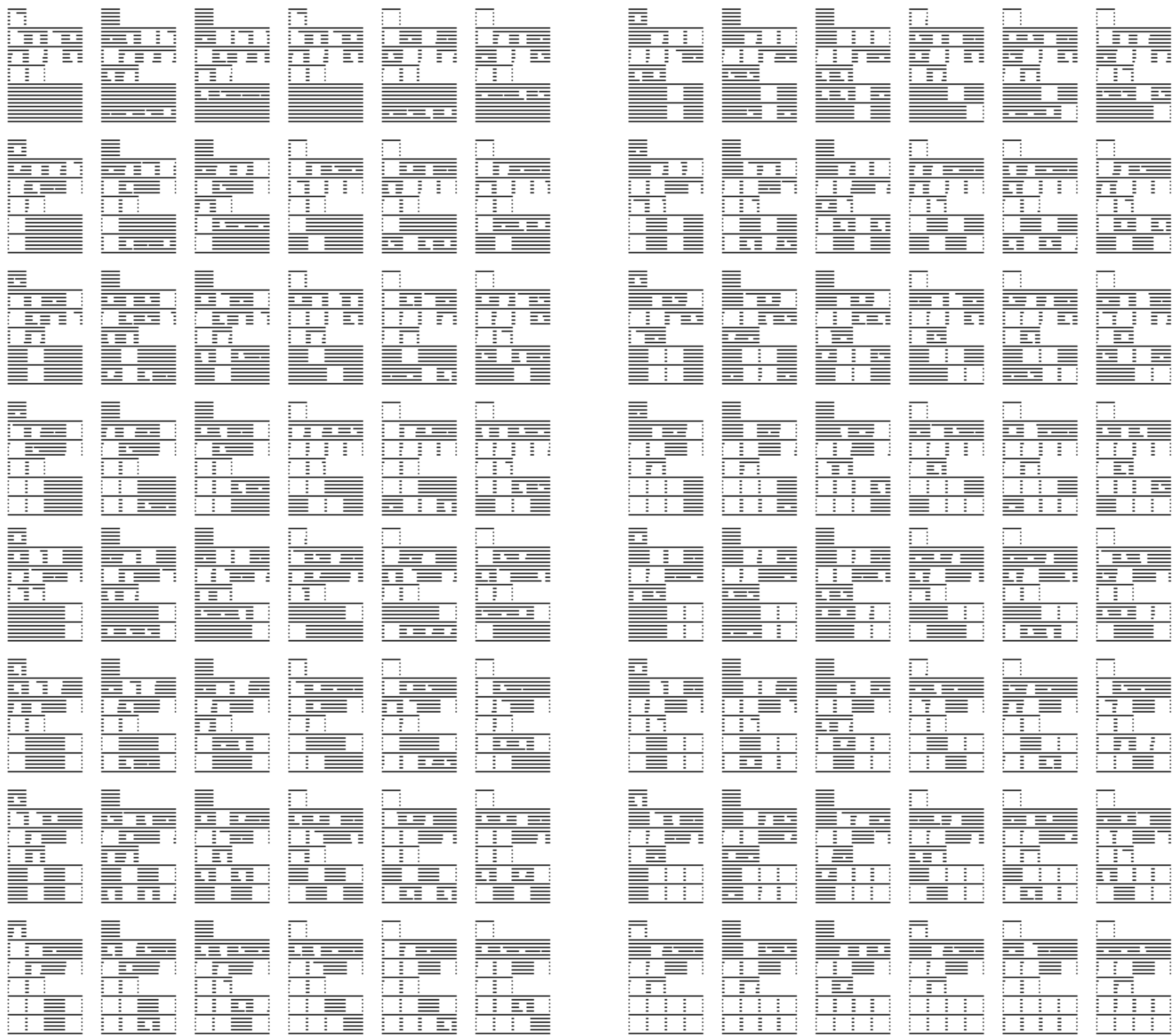

Fig. 4. Network state for all the cases in the four bit rotation problem. This display shows the states of the units, arranged as in figure 2. Each row of six shows one value for register $A$. There are $2^{4}=16$ such rows. Within each row, the three diagrams on the left show the network's state when competing the direction bit, register B, and register A, unshifted. The right three are the same, except with a shift. Note that all completions are correct except in the two cases where the rotation bit can not be determined from the two shift registers, namely a pattern of 0000 or 1111 .

values, the network is allowed to settle, and the quantities

$$
p_{i j}^{+}=\left\langle y_{i} y_{j}\right\rangle=\sum_{\alpha, \beta} P(\alpha) y_{i}^{(\alpha, \beta)} y_{j}^{(\alpha, \beta)} .
$$

are accumulated, where $\langle\cdot\rangle$ denotes an average over the environmental distribution, the + superscript denote clamping of both input and output, and $\alpha$ is used to index the input units and $\beta$ indexes the output units. The same procedure is then repeated, but with the output units (states of which we will index by $\beta$ ) not clamped, yielding

$$
p_{i j}^{-}=\left\langle y_{i} y_{j}\right\rangle=\sum_{\alpha} P(\alpha) y_{i}^{(\alpha)} y_{j}^{(\alpha)}
$$

where the - superscript denotes clamping of only the inputs and not the outputs. At this point, it is the case that

$$
\frac{\partial G}{\partial w_{i j}}=p_{i j}^{+}-p_{i j}^{-}
$$

where

$$
G=\sum_{\alpha, \beta} P(\alpha) \log \frac{P(\beta \mid \alpha)}{P^{-}(\beta \mid \alpha)}
$$

is a measure of the information theoretic difference between the clamped and unclamped distribution of the output units given the clamped input units. $P^{-}(\beta \mid \alpha)$ measures how probable the network says $\beta$ is given $\alpha$, and its definition is beyond the scope of this paper, while $P(\beta \mid \alpha)$ is 
the probability of $\beta$ being the correct output when $\alpha$ is the input, as given by the target distribution to be learned.

This learning rule (14) is a version of Hebb's rule in which the sign of synaptic modification is alternated, positive during the "waking" phase and negative during the "hallucinating" phase.

Even before the learning rule was rigorously justified, deterministic Boltzmann Machines were applied to a number of tasks [92], [91]. Although weight symmetry is assumed in the definition of energy which is used in the definition of probability, and is thus fundamental to these mathematics, it seems that in practice weight asymmetry can be tolerated in large networks [88]. This makes MFT Boltzmann Machines the most biologically plausible of the various learning procedures we discuss, but it is difficult to see how it would be possible to extend them to learning more complex phenomena, like limit cycles or paths through state space. And thus, although they are probably the best current technique in their domain of application, we now turn our attention to procedures suitable for learning more dynamic sorts of behaviors.

\section{Computing the Gradient Without Assuming a Fixedpoint}

Now we get to the heart of the matter-the computation of $\nabla_{w} E$, the gradient of the error $E$ with respect to the vector of free parameters $w$, where the error is not defined at a fixedpoint but rather is a function of the network's detailed temporal behavior. The techniques we will discuss here, like those of section II, are quite general purpose: they can accommodate hidden units as well as various architectural embellishments, such as second-order connections [93], [34], [94], [44], weight sharing [23], [35], and in general any of the architectureal modifications made to neural networks to customize them for their problem domain. We will consider two major gradient calculation techniques, and then a few more derived from them. The first is the obvious extension of backpropagation through time (BPTT) to continuous time [95], [96], [62].

\section{A. Backpropagation Through Time}

The fixedpoint learning procedures discussed above are unable to learn non-fixedpoint attractors, or to produce desired temporal behavior over a bounded interval, or even to learn to reach their fixedpoints quickly. Here, we turn to a learning procedure suitable for such non-fixedpoint situations. This learning procedure essentially converts a network evolving through time into a network whose activation is flowing through a number of layers, translating time into space, as shown in figure 5. Backpropagation then becomes applicable. The technique is therefore called Backpropagation Through Time, or BPTT.

Consider minimizing $E(\mathbf{y})$, some functional of the trajectory taken by $\mathbf{y}$ between $t_{0}$ and $t_{1}$. For instance, $E=\int_{t_{0}}^{t_{1}}\left(y_{0}(t)-d(t)\right)^{2} d t$ measures the deviation of $y_{0}(t)$ from the function $d(t)$, and minimizing this $E$ would teach the network to have $y_{0}(t)$ imitate $d(t)$. Below, we derive a technique for computing $\partial E(\mathbf{y}) / \partial w_{i j}$ efficiently, thus al- lowing us to do gradient descent in the weights so as to minimize $E$. Backpropagation through time has been used to train discrete time networks to perform a variety of tasks [59], [89]. Here, we will derive the continuous time version of backpropagation through time, as in [96], and use it in two toy domains.

In this derivation, we take the conceptually simple approach of unfolding the continuous time network into a discrete time network with a step of $\Delta t$, applying backpropagation to this discrete time network, and taking the limit as $\Delta t$ approaches zero to get a continuous time learning rule. The derivative in (3) can be approximated with

$$
\frac{d y_{i}}{d t}(t) \approx \frac{y_{i}(t+\Delta t)-y_{i}(t)}{\Delta t}
$$

which yields a first order difference approximation to (3),

$$
\tilde{y}_{i}(t+\Delta t)=(1-\Delta t) \tilde{y}_{i}(t)+\Delta t \sigma\left(\tilde{x}_{i}(t)\right)+\Delta t I_{i}(t) .
$$

Tildes are used throughout for temporally discretized versions of continuous functions.

Let us define $e_{i}$ to be the first variation of $E$ with respect to the function $y_{i}(t)$,

$$
e_{i}(t)=\frac{\delta E}{\delta y_{i}(t)}
$$

In the usual case $E$ is of the form

$$
E=\int_{t_{0}}^{t_{1}} f(\mathbf{y}(t), t) d t
$$

so $e_{i}(t)=\partial f(\mathbf{y}(t), t) / \partial y_{i}(t)$. Intuitively, $e_{i}(t)$ measures how much a small change to $y_{i}$ at time $t$ affects $E$ if everything else is left unchanged.

As usual in backpropagation, let us define

$$
\tilde{z}_{i}(t)=\frac{\partial^{+} E}{\partial \tilde{y}_{i}(t)}
$$

where the $\partial^{+}$denotes the ordered derivative of [97], with variables ordered here by time and not unit index. Intuitively, $\tilde{z}_{i}(t)$ measures how much a small change to $\tilde{y}_{i}$ at time $t$ affects $E$ when this change is propagated forward through time and influences the remainder of the trajectory, as in figure 7 . Of course, $z_{i}$ is the limit of $\tilde{z}_{i}$ as $\Delta t \rightarrow 0$. This $z$ is the $\delta$ of the standard backpropagation "generalized $\delta$ rule."

We can use the chain rule for ordered derivatives to calculate $\tilde{z}_{i}(t)$ in terms of the $\tilde{z}_{j}(t+\Delta t)$. According to the chain rule, we add all the separate influences that varying $\tilde{y}_{i}(t)$ has on $E$. It has a direct contribution of $\Delta t e_{i}(t)$, which comprises the first term of our equation for $\tilde{z}_{i}(t)$. Varying $\tilde{y}_{i}(t)$ by $d \tilde{y}_{i}(t)$ has an effect on $\tilde{y}_{i}(t+\Delta t)$ of $d \tilde{y}_{i}(t)(1-\Delta t)$, giving us a second term, namely $(1-\Delta t) \tilde{z}(t+\Delta t)$.

Each weight $w_{i j}$ makes $\tilde{y}_{i}(t)$ influence $\tilde{y}_{j}(t+\Delta t), i \neq$ $j$. Let us compute this influence in stages. Varying $\tilde{y}_{i}(t)$ by $d \tilde{y}_{i}(t)$ varies $\tilde{x}_{j}(t)$ by $d \tilde{y}_{i}(t) w_{i j}$, which varies $\sigma\left(\tilde{x}_{j}(t)\right)$ by $d \tilde{y}_{i}(t) w_{i j} \sigma^{\prime}\left(\tilde{x}_{j}(t)\right)$, which varies $\tilde{y}_{j}(t+\Delta t)$ 

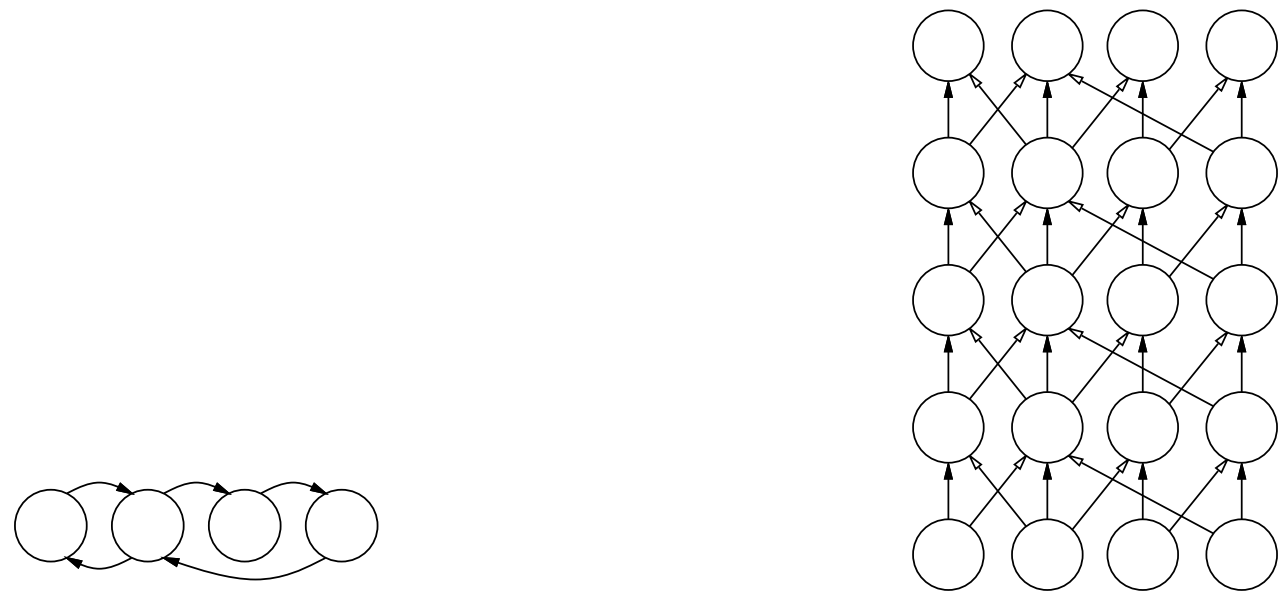

Fig. 5. A recurrent network is shown on the left, and a representation of that network unfolded in time through four time steps is shown on the right.

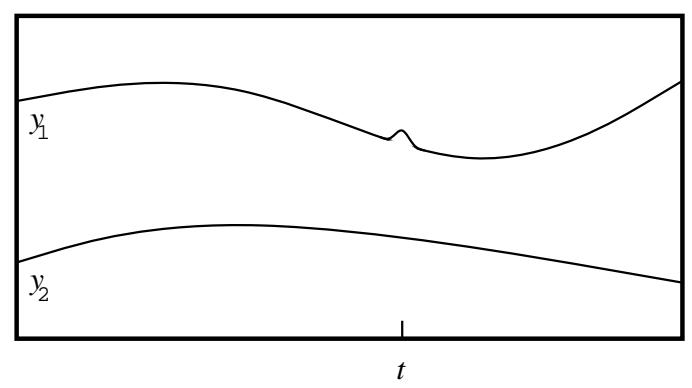

Fig. 6. The infinitesimal changes to $\mathrm{y}$ considered in $e_{1}(t)$.

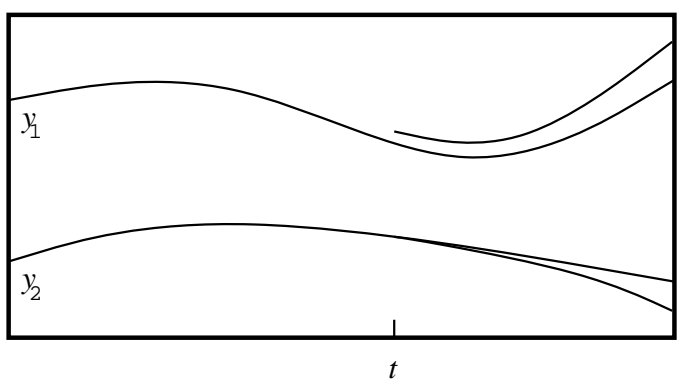

Fig. 7. The infinitesimal changes to $\mathrm{y}$ considered in $z_{1}(t)$. by $d \tilde{y}_{i}(t) w_{i j} \sigma^{\prime}\left(\tilde{x}_{j}(t)\right) \Delta t$. This gives us our third and final term, $\sum_{j} w_{i j} \sigma^{\prime}\left(\tilde{x}_{j}(t)\right) \Delta t \tilde{z}_{j}(t+\Delta t)$. Combining these,

$\tilde{z}_{i}(t)=\Delta t e_{i}(t)+(1-\Delta t) \tilde{z}_{i}(t+\Delta t)+\sum_{j} w_{i j} \sigma^{\prime}\left(\tilde{x}_{j}(t)\right) \Delta t \tilde{z}_{j}(t+\Delta t)$.

If we put this in the form of (16) and take the limit as $\Delta t \rightarrow 0$ we obtain the differential equation

$$
\begin{aligned}
\frac{d z}{d t} & =\frac{d f(y, w, I)}{d y} z+\frac{\delta E}{\delta y} \\
\frac{d E}{d w} & =\int_{t_{0}}^{t_{1}} y \frac{d f(y, w, I)}{d w} z d t .
\end{aligned}
$$

with boundary condition $z\left(t_{1}\right)=0$. Thus we have derived appropriate adjoint equations to (1). They are similar to the analogous discrete-time backwards error equations,

$$
\begin{aligned}
z(t-1) & =\frac{d f(y, w, I)}{d y} z+\frac{\partial E}{\partial y(t)} \\
\frac{d E}{d w} & =\sum_{t} y \frac{d f(y, w, I)}{d w} z .
\end{aligned}
$$

where the error to be minimized is $E$. If this error is of the usual form of an integral $E=\int E^{\prime}(y(t), t) d t$ then we get the simple form $\delta E / \delta y=d E^{\prime} / d y$.
For the particular form of (3), this comes to

$$
\frac{d z_{i}}{d t}=z_{i}-e_{i}-\sum_{j} w_{i j} \sigma^{\prime}\left(x_{j}\right) z_{j} .
$$

For boundary conditions note that by (18) and (20) $\tilde{z}_{i}\left(t_{1}\right)=\Delta t \epsilon_{i}\left(t_{1}\right)$, so in the limit as $\Delta t \rightarrow 0$ we have $z_{i}\left(t_{1}\right)=0$.

Consider making an infinitesimal change $d w_{i j}$ to $w_{i j}$ for a period $\Delta t$ starting at $t$. This will cause a corresponding infinitesimal change in $E$ of $y_{i}(t) \sigma^{\prime}\left(x_{j}(t)\right) \Delta t z_{j}(t) d w_{i j}$. Since we wish to know the effect of making this infinitesimal change to $w_{i j}$ throughout time, we integrate over the entire interval, yielding

$$
\frac{\partial E}{\partial w_{i j}}=\int_{t_{0}}^{t_{1}} y_{i} \sigma^{\prime}\left(x_{j}\right) z_{j} d t .
$$

One can also derive (26), (27) and (37) using the calculus of variations and Lagrange multipliers, as in optimal control theory [98], [99]. In fact, the idea of using gradient descent to optimize complex systems was explored by control theorists in the late 1950s. Although their mathematical techniques and algorithms are identical to those reviewed here, and thus handled hidden units, they refrained from exploring systems with so many degrees of freedom, perhaps in fear of local minima.

It is also interesting to note that the recurrent backpropagation learning rule (section II-C) can be derived from 
these. Let $I_{i}$ be held constant, assume that the network settles to a fixedpoint, and let $E$ be integrated for one time unit before $t_{1}$. As $t_{1} \rightarrow \infty,(26)$ and (27) reduce to the recurrent backpropagation equations (9) and (8), so in this sense backpropagation through time is a generalization of recurrent backpropagation.

There are two ways to go about finding such derivations. One is direct, using the calculus of variations [98]. The other is to take the continuous time equations, approximate them by difference equations, precisely calculate the adjoint equations for this discrete time system, and then approximate back to get the continuous time adjoint equations, as in [76]. An advantage of the latter approach is that, when simulating on a digital computer, one actually simulates the difference equations. The derivation ensures that the simulated adjoint difference equations are the precise adjoints to the simulated forward difference equations, so the computed derivatives contain no approximation errors.

\section{B. Real Time Recurrent Learning}

An online, exact, and stable, but computationally expensive, procedure for determining the derivatives of functions of the states of a dynamic system with respect to that system's internal parameters has been discovered and applied to recurrent neural networks a number of times [100], [101], [102], [103]; for reviews see also [81], [76], [104]. It is called by various researchers forward propagation, forward perturbation, or real time recurrent learning, RTRL. Like BPTT, the technique was known and applied to other sorts of systems since the 1950s; for a hook into this literature see [105], [106] or the closely related Extended Kalman Filter [107]. In the general case of (1), RTRL is

$$
\frac{d E}{d w}=\int_{t_{0}}^{t_{1}} \gamma \frac{\delta E}{\delta y} d t
$$

where $\gamma\left(t_{0}\right)=0$ and

$$
\frac{d \gamma}{d t}=\frac{d f(y, w, I)}{d w}+\frac{d f(y, w, I)}{d y} \gamma
$$

The $\gamma$ matrix is the sensitivity of the states $y(t)$ to a change of the weights $w$.

Under the assumption that the weights are changing slowly, RTRL can be made an online algorithm by updating the weights continuously instead of actually integrating (28),

$$
\frac{d w}{d t}=-\eta \gamma \frac{\delta E}{\delta y},
$$

where $\eta$ is the learning rate, or, if a momentum term $0<$ $\alpha<1$ is also desired,

$$
\alpha \frac{d^{2} w}{d t^{2}}+(1-\alpha) \frac{d w}{d t}+\eta \gamma \frac{\delta E}{\delta y}=0
$$

For the special case of a fully connected recurrent neural network, as described by (3), applying the general RTRL formulas above yields

$$
\begin{array}{r}
\frac{d \gamma_{i j k}}{d t}=\frac{\partial f_{k}}{\partial y_{k}} \gamma_{i j k}+\left([j=k] y_{j}+\sum_{l} w_{l k} \gamma_{i j l}\right) \frac{\partial f_{k}}{\partial n e t_{k}} \\
\frac{d w_{i j}}{d t}(t)=-\eta \sum_{k} \frac{\partial g}{\partial y_{k}}(t) \gamma_{i j k}(t)
\end{array}
$$

Regretably, the computation of $\gamma$ is very expensive, and also non-local. The $\gamma$ array has $n m$ elements, where $n$ is the number of states and $m$ the number of weights, which is typically on the order of $n^{2}$. Updating $\gamma$ requires $O\left(n^{3} m\right)$ operations in the general case, but the particular structure of a neural network causes some of the matrices to be sparse, which reduces the burden to $O\left(n^{2} m\right)$. This remain too high to make the technique practical for large networks. Nevertheless, because of its ease of implementation, RTRL is used by many researchers working with small networks.

\section{Less Computationally Burdensome Online Techniques}

One way to reduce the complexity of the RTRL algorithm is to simply leave out elements of $\gamma$ that one has reason to believe will remain approximately zero. This approach, in particular ignoring the coupling terms which relate the states of units in one module to weights in another, has been explored by Zipser [108].

Another is to use BPTT with a history cutoff of $k$ units of time, termed BPTT $(k)$ by Williams and Peng [109], and make a small weight change each timestep. This obviates the need for epochs, resulting in a purely online technique, and is probably the best technique for most practical problems.

A third is to take blocks of $s$ timesteps using BPTT, but use RTRL to encapsulate the history before the start of each block. This requires $O\left(s^{-1} n^{2} m+n m\right)$ time per step, on average, and $O(n m+s m)$ space. Choosing $s=n$ makes this $O(\mathrm{~nm})$ time and $O(\mathrm{~nm})$ space, which dominates RTRL. This technique has been discovered independently a number of times [110], [111].

Finally, one can note that, although the forward equations for $y$ are nonlinear, and therefore require numeric integration, the backwards equations for $z$ in BPTT are linear. Since the $d E / d w$ terms are linear integrations of the $z$, this means that they are linear functions of the external inputs, namely the $e_{i}$ terms. As shown by Sun et al. [112], this allows one, during the forward pass, to compute a matrix relating the external error signal to the elements of $\nabla_{w}$, allowing a fully online algorithm with $O(\mathrm{~nm})$ time and space complexity.

\section{Time Constants}

A major advantage of temporally continuous networks is that one can add additional parameters that control the temporal bahavior in ways known to relate to natural tasks. An example of this is time constants, which were learned in the context of neural networks in [79], [53], [52]. If we add a time constant $T_{i}$ to each unit $i$, modifying (3) to

$$
T_{i} \frac{d y_{i}}{d t}=-y_{i}+\sigma\left(x_{i}\right)+I_{i}
$$


and carry these terms through the derivation of section IIIA, equations (26) and (27) become

$$
\frac{d z_{i}}{d t}=\frac{1}{T_{i}} z_{i}-e_{i}-\sum_{j} \frac{1}{T_{j}} w_{i j} \sigma^{\prime}\left(x_{j}\right) z_{j} .
$$

and

$$
\frac{\partial E}{\partial w_{i j}}=\frac{1}{T_{j}} \int_{t_{0}}^{t_{1}} y_{i} \sigma^{\prime}\left(x_{j}\right) z_{j} d t
$$

In order to learn these time constants rather than just set them by hand, we need to compute $\partial E(\mathbf{y}) / \partial T_{i}$. If we substitute $\rho_{i}=T_{i}^{-1}$ into (34), find $\partial E / \partial \rho_{i}$ with a derivation similar to that of (27), and substitute $T_{i}$ back in we get

$$
\frac{\partial E}{\partial T_{i}}=-\frac{1}{T_{i}} \int_{t_{0}}^{t_{1}} z_{i} \frac{d y_{i}}{d t} d t
$$

\section{E. Time Delays}

Consider a network in which signals take finite time to travel over each link, so that (4) is modified to

$$
x_{i}(t)=\sum_{j} w_{j i} y_{j}\left(t-\tau_{j i}\right)
$$

$\tau_{j i}$ being the time delay along the connection from unit $j$ to unit $i$. Let us include the variable time constants of section III-D as well. Such time delays merely add analogous time delays to (35) and (36),

$$
\begin{gathered}
\frac{d z_{i}}{d t}(t)=\frac{1}{T_{i}} z_{i}(t)-e_{i}(t)-\sum_{j} w_{i j} \sigma^{\prime}\left(x_{j}\left(t+\tau_{i j}\right)\right) \frac{1}{T_{j}} z_{j}\left(t+\tau_{i j}\right) \\
\frac{\partial E}{\partial w_{i j}}=\frac{1}{T_{j}} \int_{t_{0}}^{t_{1}} y_{i}(t) \sigma^{\prime}\left(x_{j}\left(t+\tau_{i j}\right)\right) z_{j}\left(t+\tau_{i j}\right) d t
\end{gathered}
$$

while (37) remains unchanged. If we set $\tau_{i j}=\Delta t$, these modified equations alleviate concern over time skew when simulating networks of this sort, obviating any need for accurate numerical simulations of the differential equations and allowing simple difference equations to be used without fear of inaccurate error derivatives.

Instead of regarding the time delays as a fixed part of the architecture, we can imagine modifiable time delays. Given modifiable time delays, we would like to be able to learn appropriate values for them, which can be accomplished using gradient descent by

$$
\frac{\partial E}{\partial \tau_{i j}}=\int_{t_{0}}^{t_{1}} z_{j}(t) \sigma^{\prime}\left(x_{j}(t)\right) w_{i j} \frac{d y_{i}}{d t}\left(t-\tau_{i j}\right) d t
$$

[12] applied recurrent networks with immutable time delays in the domain of speech. Feedforward networks with immutable time delays (TDNNs) have been applied with great success in the same domain by Lang et al. [22]. A variant of TDNNs which learn the time delays was explored by Bodenhausen et al. [113]. The synapses in their networks, rather than having point taps, have gaussian envelopes whose widths and centers were both learned. Similar synaptic architectures using alpha function envelopes (which obviate the need for a history buffer) whose parameters were learned were proposed and used in systems without hidden units [114], [29]. A continuous time feedforward network with learned time delays was successfully applied to a difficult time-series prediction task by Day and Davenport [25].

In the sections on time constants and delays, we have carried out the derivative derivations for BPTT. All the other techniques also remain applicable to this case, with straightforward derivations. The analogous derivations for RTRL are carried out in [76]. However, we will not here simulate networks with modifiable time delays.

An interesting class of architectures would have the state of one unit modulate the time delay along some arbitrary link in the network or the time constant of some other unit. Such a "higher order time delay" architecture seems appropriate for tasks in which time warping is an issue, such as speech recognition. The gradients with respect to higher order time delay can be readily calculated by appropriate augmentation of either BPTT or RTRL.

In the presence of time delays, it is reasonable to have more than one connection between a single pair of units, with different time delays along the different connections. Such "time delay neural networks" have proven useful in the domain of speech recognition [20], [22], [21], [115]. Having more than one connection from one unit to another requires us to modify our notation somewhat; weights and time delays are modified to take a single index, and we introduce some external apparatus to specify the source and destination of each connection. Thus $w_{i}$ is the weight on a connection between unit $\mathcal{L}(i)$ and unit $\mathcal{R}(i)$, and $\tau_{i}$ is the time delay along that connection. Using this notation we write $(38)$ as

$$
x_{i}(t)=\sum_{j \mid \mathcal{L}(j)=i} w_{j} y_{\mathcal{R}(j)}\left(t-\tau_{j}\right) .
$$

Our equations would be more general if written in this notation, but readability would suffer, and the translation is quite mechanical.

\section{F. Extending RTRL to Time Constants and Time Delays}

We have seen that BPTT can be easily applied to these new sorts of free parameters we have been adding to our networks, namely time constants and time delays. Other gradient calculation procedures also can be naturally applied to these new sorts of free parameters. In this section, we apply RTRL, first to incorporate time constants and then time delays.

If we begin with (34), first we must generalize (32) and (33) to correctly modify the weights in the presence of time constants. If we substitute $k$ for $i$ in (34), take the partial with respect to $w_{i j}$, and substitute in $\gamma$ where possible, we have a the differential equation for $\gamma$

$$
T_{k} \frac{\gamma k i j}{d t}=-\gamma k i j+\sigma^{\prime}\left(x_{k}\right) \sum_{l} w_{l k} \gamma l i j
$$

nearly the same as (32) except for a time constant. 
We can derive analogous equations for the time constants themselves; define

$$
q_{j}^{i}(t)=\frac{\partial y_{i}(t)}{\partial T_{j}}
$$

take the partial of (3) with respect to $T_{j}$, and substitute in $q$. This yields

$$
T_{i} \frac{d q_{j}^{i}}{d t}=-q_{j}^{i}-\frac{d y_{i}}{d t}+\sigma^{\prime}\left(x_{i}\right) \sum_{k} w_{k i} q_{j}^{k}
$$

which can be used to update the time constants using the continuous update rule

$$
\frac{d T_{i}}{d t}=-\eta \sum_{j} e_{j} q_{i}^{j}
$$

Similarly, let us derive equations for modifying the time delays of section III-E. Define

$$
r_{i j}^{k}(t)=\frac{\partial y_{k}(t)}{\partial \tau_{i j}}
$$

and take the partial of (3) with respect to $\tau_{i j}$, arriving at a differential equations for $r$,

$$
T_{k} \frac{d r_{i j}^{k}}{d t}=-r_{i j}^{k}+\sigma^{\prime}\left(x_{k}\right)(\underbrace{w_{i j} \frac{d y_{i}}{d t}\left(t-\tau_{i j}\right)}_{\text {included if } j=k}-\sum_{l} w_{l k} r_{i j}^{l}\left(t-\tau_{l k}\right)) .
$$

The time delays can be updated online using the continuous update equation

$$
\frac{d \tau_{i j}}{d t}=-\eta \sum_{k} e_{k} r_{i j}^{k}
$$

\section{Some Simulations}

In the following simulations, we used networks without time delays, but with mutable time constants. As in the associative network of section II-C.1, an extra input unit whose value was always held at 1 by a constant external input of 0.5 , and which had outgoing connections to all other units, was used to implement biases.

Using first order finite difference approximations, we integrated the system $\mathbf{y}$ forward from $t_{0}$ to $t_{1}$, set the boundary conditions $z_{i}\left(t_{1}\right)=0$, and integrated the system $\mathbf{z}$ backwards from $t_{1}$ to $t_{0}$ while numerically integrat$\operatorname{ing} z_{j} \sigma^{\prime}\left(x_{j}\right) y_{i}$ and $z_{i} d y_{i} / d t$, thus computing $\partial E / \partial w_{i j}$ and $\partial E / \partial T_{i}$. Since computing $d z_{i} / d t$ requires $\sigma^{\prime}\left(x_{i}\right)$, we stored it and replayed it backwards as well. We also stored and replayed $y_{i}$ as it is used in expressions being numerically integrated.

We used the error functional

$$
E=\frac{1}{2} \sum_{i} \int_{t_{0}}^{t_{1}} s_{i}\left(y_{i}-d_{i}\right)^{2} d t
$$

where $d_{i}(t)$ is the desired state of unit $i$ at time $t$ and $s_{i}(t)$ is the importance of unit $i$ achieving that state at that time, in this case 0 except when $i$ was an output unit and after some time (5 units) had elapsed for the network to settle down. Throughout, we used $\sigma(\xi)=\left(1+\epsilon^{-\xi}\right)^{-1}$. Time constants were initialized to 1 , weights were initialized to uniformly distributed random values between 1 and -1 , and the initial values $y_{i}\left(t_{0}\right)$ were set to $I_{i}\left(t_{0}\right)+\sigma(0)$. The simulator used first order difference equations (17) and (21) with $\Delta t=0.1$.

\section{A. A Rotated Figure Eight}

In this simulation a network was trained to generate a figure eight shaped trajectory in two of its units, designated output units. The figure eight was to be rotated about its center by an angle $\theta$ which was input to the network through two input units which held the coordinates of a unit vector in the appropriate direction. This was intended to model a controlled modulation of a central pattern generator from tonic modulatory input, as in the lobster stomatagastric gangleon [116]. The target vector for the two output units was generated by

target $=0.4\left(\begin{array}{cc}\cos \theta & -\sin \theta \\ \sin \theta & \cos \theta\end{array}\right)\left(\begin{array}{c}\sin \pi t / 16 \\ \cos \pi t / 16\end{array}\right)+\left(\begin{array}{c}0.5 \\ 0.5\end{array}\right)$

while the input to the network was simply the angle $\theta$, represented to avoid blemishes as the direction vector

$$
\left(\begin{array}{c}
\sin \theta \\
\cos \theta
\end{array}\right)
$$

Eight different values of $\theta$, equally spaced about the circle, were used to generate the training data. In experiments with 20 hidden units, the network was unable to learn the task. Increasing the number of hidden units to 30 allowed the network to learn the task, as shown on the left in figure 8 . But as shown on the right in figure 8 , generalization is poor when the network is run with the eight input angles furthest from the training angles, i.e. 22.5 degrees off.

The task would be simple to solve using second order connections, as they would allow the problem to be decoupled. A few units could be devoted to each of the orthogonal oscillations, and the connections could form a rotation matrix. The poor generalization of the network shows that it is not solving the problem in such a straightforward fashion, and suggests that for tasks of this sort it might be better to use slightly higher order units.

\section{Stability and Perturbation Experiments}

We can analytically determine the stability of the network by measuring the eigenvalues of $D f$ where $f$ is the function that maps the state of the network at one point in time to its state at a later time. For instance, for a network exhibiting a limit cycle one would typically use the function that maps the network's state at some time in the cycle to its state at the corresponding time in the next cycle. Unfortunately, this gives only a local stability measure, and also does not factor out the effect of hidden units. 

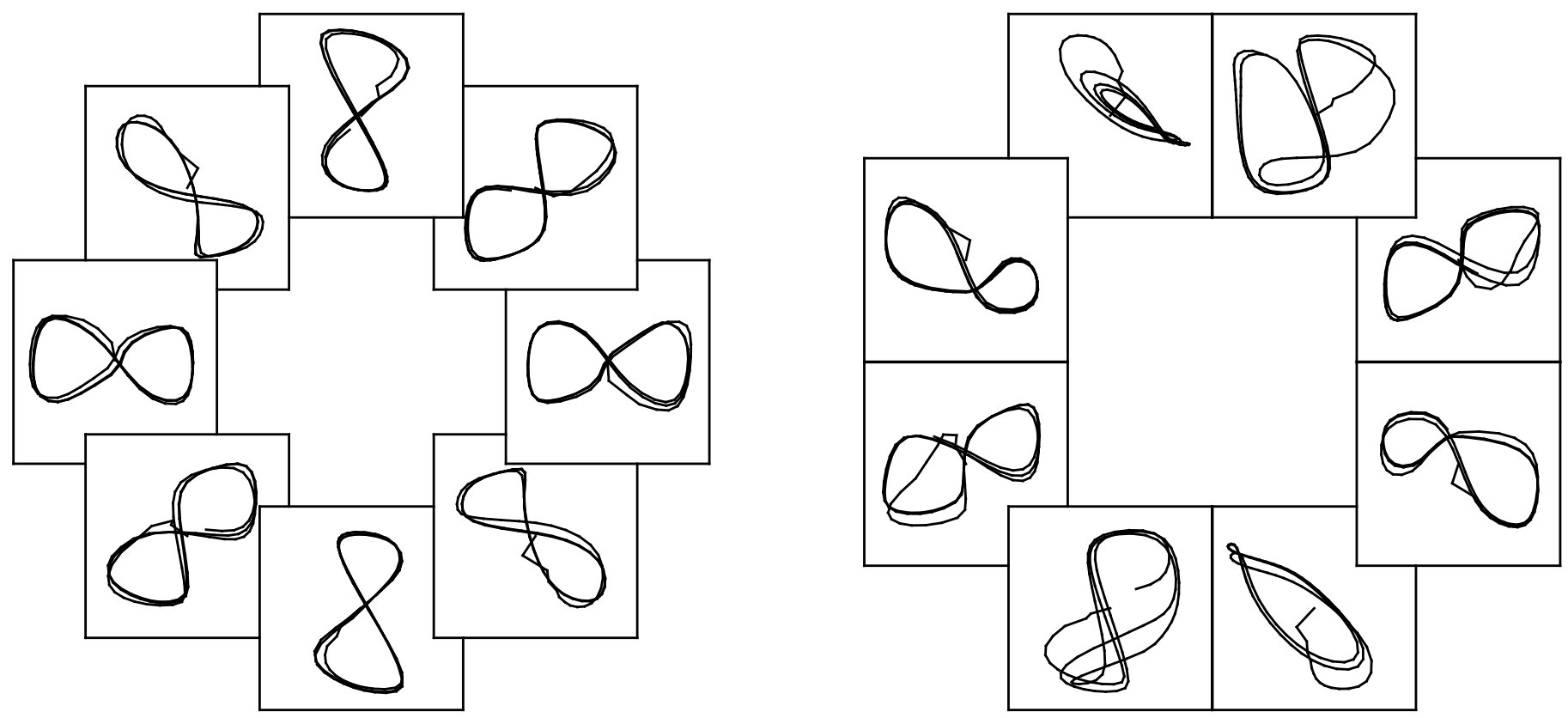

Fig. 8. The output of the rotated figure eight network at all the trained angles (left) and some untrained angles (right).

In our attempt to judge the stability of the limit cycles exhibited above, rather than calculating $D f$, where $f(y(t))=y(t+16)$, we simply modified the simulator to introduce random perturbations and observed the effects of these perturbations upon the evolution of the system. ${ }^{4}$ The two output units in the unrotated figure eight task appear to be phase locked, as their phase relationship remains invariant even in the face of major perturbations. This phase locking is unlike the solution that a human would create by analytically determining weights through decoupling the two output units and using linearized subnets to generate the desired oscillatory behavior, as suggested by Merrick Furst.

The networks to which we introduced these perturbations had been trained to produce simple limit cycles, one in a circular shape, and the other in a figure eight shape. Neither of the networks had any input units; they produced only a single limit cycle.

The unperturbed limit cycle of the figure eight network is symmetric, but when perturbations are introduced, as in the right of figure 9 , symmetry is broken. The portion of the limit cycle moving from the upper left hand corner towards the lower right hand corner has diverging lines, but we do not believe that they indicate high eigenvalues and instability. The lines converge rapidly in the upward stroke on the right hand side of the figure, and analogous unstable behavior is not present in the symmetric downward stroke from the upper right hand corner towards the lower left. Analysis shows that the instability is caused by the initialization circuitry being inappropriately activated. Since the

\footnotetext{
${ }^{4}$ Actually, we wouldn't care about the eigenvalues of $D f$ per se, because we wouldn't care about perturbations in the direction of travel, as these effect only the phase, or perturbations that effect only the hidden units. For this reason, we would want to project these out of the matrix $D f$ before computing the eigenvalues. This effect is achieved automatically in our display in figure 9.
}

initialization circuitry is adapted for controlling just the initial behavior of the network, when the net must delay at $(0.5,0.5)$ for a time before beginning the cycle by moving towards the lower left corner, this circuitry is explicitly not symmetric. The diverging lines seem to be caused by this circuitry being activated and exerting a strong influence on the output units while the circuitry itself deactivates.

In fact, [117] developed a technique for learning the local maximum eigenvalue of the transfer function, optionally projecting out directions whose eigenvalues are not of interest. This technique, which explicitly modulates the behavior we only measured above, has not yet been applied in a control domain.

\section{Other Non-FIXEDPoint TeChNiQues}

\section{A. "Elman Nets"}

[118] considers a version of backpropagation through time in discrete time in which the temporal history is cut off. Typically, only one or two timesteps are preserved, at the discretion of the architect. This cutoff makes backpropagation through time an online algorithm, as the backpropagation to be done to account for the error at each point in time is done immediately. However, it makes the computational expense per time step scale linearly with the number of timesteps of history being maintained. This accuracy of the computed derivative is smoothly traded off against storage and computation.

The real question with Elman networks is whether the contribution to the error from the history that has been cut off is significant. This question can only be answered relative to a particular task. For instance, [119] finds some problems amenable to the history cutoff, but resorts to full fledged backpropagation through time for other tasks. [43] describe a regular language token prediction task which is difficult for Elman nets when the transition probabili- 

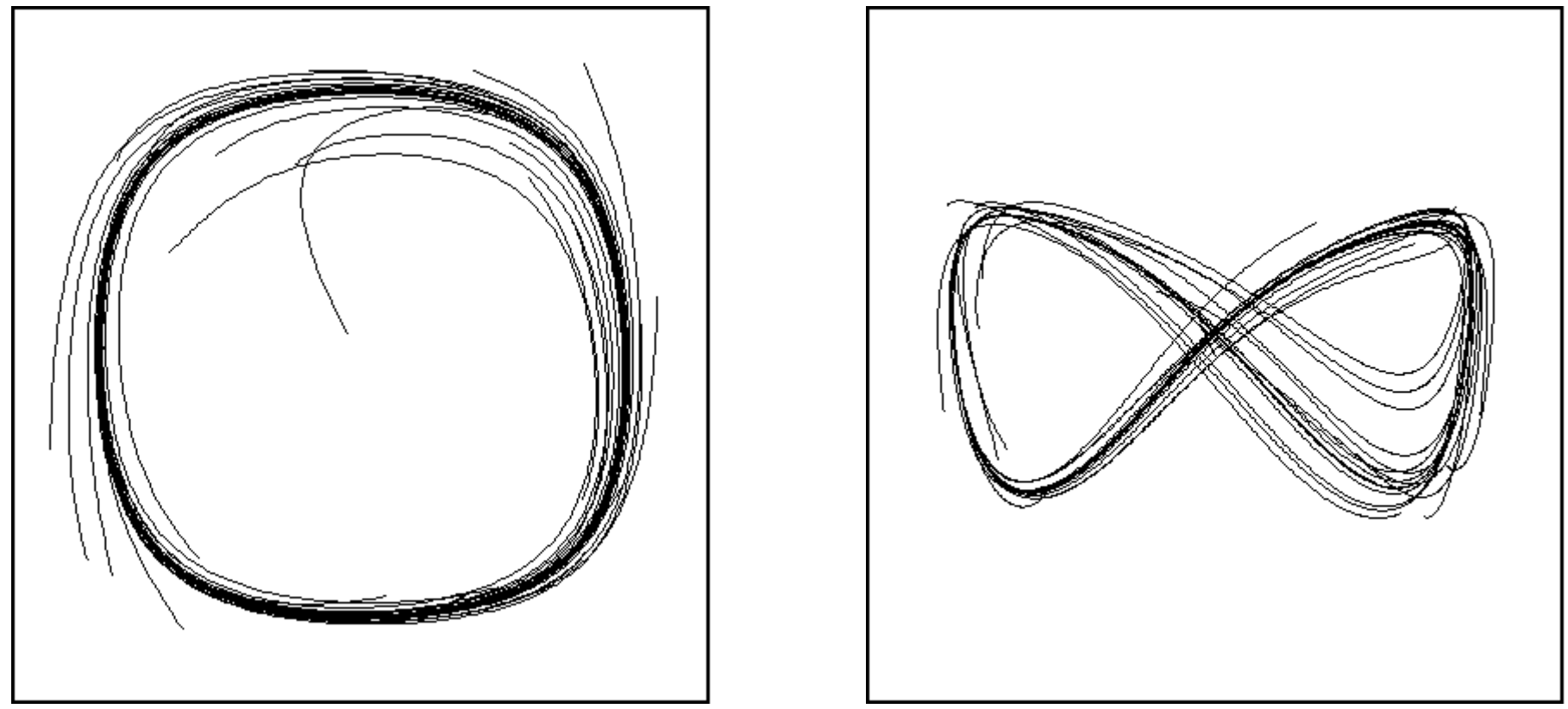

Fig. 9. The output states $y_{1}$ and $y_{2}$ plotted against each other for a 1000 time unit run, with all the units in the network perturbed by a random amount about every 40 units of time. The perturbations in the circle network (left) were uniform in \pm 0.1 , and in the figure eight network (right) in \pm 0.05 .

ties are equal, but find that breaking this symmetry allows these nets to learn the task.

\section{B. The Moving Targets Method}

[120], [121], [122] propose a moving targets learning algorithm. Such an algorithm maintains a target value for each hidden unit at each point in time. These target values are typically initialized either randomly, or to the units' initial untrained behavior. In learning, two phases alternate. In one phase, the hidden units' targets are improved, such that if the targets are attained better performance would be achieved. In the other phase, the weights are modified such that each unit comes closer to attaining its target values. The error can be regarded as having two terms, one term which penalizes the units being too far from their targets, and another which penalizes the targets for being too far from the values actually attained. This technique has the appeal of decoupling temporally distant actions during the learning of weights, and the disadvantage of requiring the targets to be stored and updated. In the limit, as learning rates are decreased, the moving targets method becomes equivalent to backpropagation though time.

In continuous time, the moving targets method would entail decoupling the units during learning, and storing a target trajectory for each unit, including the hidden units. The weights would then be modified to make the trajectories consistent with each other, while the trajectories of the hidden units would be similarly modified. Unfortunately, as with teacher forcing, even if the error is driven to very low levels by such a procedure, there would be no guarantee that the resulting network, if allowed to run free, would have dynamics close to that of the forced dynamics.

The primary disadvantage of the technique is that each pattern to be learned must have associated with it the targets for the hidden units, and these targets must be learned just as the weights are. This makes the technique inapplicable for online learning, in which each pattern is seen only once.

\section{Feedforward Networks with State}

It is noteworthy that that the same basic mathematical technique of forward propagation can be applied to networks with a restricted architecture, feedforward networks whose units have state [77], [78], [80]. This is the same as requiring the $w_{i j}$ matrix to be triangular, but allowing non-zero diagonal terms. If we let the $\gamma$ quantities be ordered derivatives, as in standard backpropagation, then this simplified architecture reduces the computational burden substantially. The elimination of almost all temporal interaction makes $\gamma_{i j k}=0$ unless $i=k$, leaving only $O\left(n^{2}\right)$ auxiliary equations, each of which can be updated with $O(1)$ computation, for a total update burden of $O\left(n^{2}\right)$, which is the same as conventional backpropagation. This favorable computational complexity makes it of practical significance even for large feedforward recurrent networks. But these feedforward networks are outside the scope of this paper.

\section{Teacher Forcing In Continuous Time}

[123] coin the term teacher forcing, which consists of jamming the desired output values into output units as the network runs. Thus, the teacher forces the output units to have the correct states, even as the network runs, and hence the name. This technique is applied to discrete time clocked networks, as only then does the concept of changing the state of an output unit each time step make sense. 
The error is as usual, with the caveat that errors are to be measured before output units are forced, not after. [123] report that their teacher forcing technique radically reduced training time for their recurrent networks, although $[76]$ reports difficulties when teacher forcing was used networks with a larger number of hidden units.

Williams and Zipser's application of teacher forcing to their networks is dependent on discrete time steps, so applying teacher forcing to temporally continuous networks requires a different approach. The approach we shall take is to add some controls that one imagines being used to control the states of the output units, and use them to keep the output units locked at their desired states. The error function to be minimized will measure the amount of control that it was necessary to exert, with zero error coming only when the no external forces at all need to be exerted.

Let

$$
F_{i}=\frac{1}{T_{i}}\left(-y_{i}+\sigma\left(x_{i}\right)+I_{i}\right)
$$

so that $(3)$ is just $d y_{i} / d t=F_{i}$, and let us add a new forcing term $f_{i}(t)$ to $(3)$,

$$
\frac{d y_{i}}{d t}=F_{i}+f_{i}
$$

Using $\Phi$ to denote the set of units to be forced, we will let $d_{i}$ be the trajectory that we will force $y_{i}$ to follow, for each $i \in \Phi$. So we set

$$
f_{i}=\frac{d d_{i}}{d t}-F_{i}
$$

and $y_{i}\left(t_{0}\right)=d_{i}\left(t_{0}\right)$ for $i \in \Phi$ and $f_{i}=0$ for $i \notin \Phi$, with the consequence that $y_{i}=d_{i}$ for $i \in \Phi$. Now let the error functional be of the form

$$
E=\int_{t_{0}}^{t_{1}} L(f(t), t) d t
$$

where typically $L=\sum_{i \in \Phi} f_{i}^{2}$.

We can modify the derivation in section III-A for this teacher forced system. For $i \in \Phi$ a change to $\tilde{y}_{i}$ will be canceled immediately, so taking the limit as $\Delta t \rightarrow 0$ yields $z_{i}=0$. Because of this, it doesn't matter what $e_{i}$ is for $i \in \Phi$.

We can apply (18) to calculate $e_{i}$ for $i \notin \Phi$. The chain rule is used to calculate how a change in $y_{i}$ effects $E$ through the $f_{i}$, yielding

$$
e_{i}=\sum_{j \in \Phi} \frac{\delta E}{\delta f_{j}} \frac{\partial f_{j}}{\partial y_{i}}
$$

or

$$
e_{i}=\sum_{j \in \Phi} \frac{\partial L}{\partial f_{j}}-\frac{1}{T_{j}} \sigma^{\prime}\left(x_{j}\right) w_{i j}
$$

For $i \notin \Phi(26)$ and (37) are unchanged, and for $j \notin \Phi$ and any $i(27)$ also remains unchanged. The only equations still required are $\partial E / \partial w_{i j}$ for $j \in \Phi$ and $\partial E / \partial T_{i}$ for $i \in \Phi$. To derive the first, consider the instantaneous effect of a small change to $w_{i j}$, giving

$$
\frac{\partial E}{\partial w_{i j}}=\frac{1}{T_{j}} \int_{t_{0}}^{t_{1}} y_{i} \sigma^{\prime}\left(x_{j}\right) \frac{\partial L}{\partial f_{i}} d t .
$$

Analogously, for $i \in \Phi$

$$
\frac{\partial E}{\partial T_{i}}=-\frac{1}{T_{i}} \int_{t_{0}}^{t_{1}} \frac{\partial L}{\partial f_{i}} \frac{d y_{i}}{d t} d t .
$$

We are left with a system with a number of special cases depending on whether units are in $\Phi$ or not. Interestingly, an equivalent system results if we leave $(26),(27)$, and $(37)$ unchanged except for setting $z_{i}=\partial L / \partial f_{i}$ for $i \in \Phi$ and setting all the $e_{i}=0$. It is an open question as to whether there is some other way of defining $z_{i}$ and $e_{i}$ that results in this simplification.

However, by taking the limit as the step size goes to zero, it is possible to show that the continuous time analogue of teacher forcing is to force the output states to follow desired trajectories, with the error being the difference between the derivative that the network attempts to apply to these units and the derivative of the desired trajectory. This casts light on teacher forcing in the descrete time case, which can be seen as nearly the same thing.

Regretably it also shows that teacher forcing can result in a network with a systematic bias, or a network which, although when being forced has little error, when running free rapidly drifts far from the desired trajectory, in a qualitative sense, as reported by Williams and Zipser for some cases where oscillations trained with teacher forcing exhibited radically and systematically lower frequency and amplitude when running free [123].

\section{E. Jordan Nets}

[124] used a backpropagation network with the outputs clocked back to the inputs to generate temporal sequences. Although these networks were used long before teacher forcing, from our perspective Jordan nets are simply a restricted class of teacher forced recurrent networks, in particular, discrete time networks in which the only recurrent connections emanate from output units. By teacher forcing these output units, no real recurrent paths remain, so simple backpropagation through a single time step suffices for training.

The main disadvantage of such an architecture is that state to be retained by the network across time must be manifest in the desired outputs of the network, so new persistent internal representations of temporal structures cannot be created. For instance, it would be impossible to train such networks to perform the figure eight task of just a single one of the patterns shown in figure 8 . In the usual control theory way, this difficulty can be partially alleviated by cycling back to the inputs not just the previous timestep's outputs, but also those from a small number of previous timesteps. The tradeoffs between using hidden units to encapsulate temporally hidden structure and using a temporal window of values which must contain the desired information is problem dependent, and depends in essence on how long a hidden variable can remain hidden without being manifested in the observable state variables.

It is easy to construct a continuous time Jordan network, in which the units' values are continuous in time, the output units constantly have corrected values jammed into 
them from external sources, and the only recurrent connections are from the outputs back to the inputs. Above we explored teacher forcing in the general setting of fully recurrent networks, but when applied to a Jordan network, the result is a system that is no longer truly recurrent, at least as far as learning is concerned. This is because the network maps the current visible state to the next visible state, with no other information retained in the network. For this reason, a continuous time Jordan network is precisely equivalent to training a layered network whose input is the current measured value of the signal we wish the Jordan network to learn, and whose target output is the first derivative of this signal to be learned.

\section{F. Teacher Forcing, RTRL, and the Kalman Filter}

[125], [126] have pointed out that RTRL is related to a version of the [127] filter, in the extension that allows it to apply to nonlinear systems, namely the extended Kalman filter (EKF) [128], [107], [129]. The EKF has time and space complexity of the same order as those of RTRL. One advantage of using the EKF (instead of RTRL) for learning the weights of a recurrent neural network, is that the EKF rationalizes teacher forcing: it modifies both the weights and the states on an equal basis. This solves the dilema of teacher forcing: that if the "true output" units are extra added units whose values are directly copied from those of the old output units, teacher forcing fails to maintain synchronization between the network and its teacher. The EKF does not have this problem, in that it would adjust the new extra and the old output units on an equal basis.

Another way of attempting to rationalize teacher forcing is to note that gradient descent itself generates $d E / d y$ in addition to $d E / d w$ terms. One might think this would make it natural to use $\Delta y=-\eta d E / d y$, thus treating the states on an equal basis with the weights. The problem with this, as pointed out by Ron Williams (personal communication) is that it is difficult to determine exactly what this means. Should the derivative be taken just with respect to the current states, or to their histories too? One way alleviate this dilema is to note that, when we change the weights, we wish we had changed them earlier. To this end, it would be natural to change the states to what they would have been had we changed the weights earlier. This gives

$$
\Delta y=\frac{d y}{d w} \Delta w
$$

The involved matrix, $d y / d w$, is already available as $\gamma$ in RTRL.

\section{Learning with Scale Parameters}

The parameters usually modified by neural network learning algorithms are the weights. There are no a priori restrictions on these values; they can be positive, negative, or zero, and the behavior of a network is continuous with respect to changes in its weights. These factors, along with the tractable shape of the error surface, make simple gradient descent algorithms, $\Delta w=-\eta d E / d w$, surprisingly effective.
The error term $E$ being used generally contains one term which has to do with how well the network's outputs meet some criterion. Frequently another term is added as an expression of some a priori known probability distribtion of the weights. For instance, adding $\sum_{i} w_{i}^{2}$ is equivalent to assuming that the weights are Gaussian distributed. Not adding such a term is equivalent to assuming that the $a$ priori distribution on what the weights will turn out to be is flat-not a totally unreasonable prior [28], [130].

However, we have added some new sorts of parameters, namely time constants and time delays, here represented generically by the vector $T$. These are scale parameters, which differ from positional parameters in a number of ways. The most telling property of a scale parameter is that the dynamics of the system are affected about as much by multiplying a scale parameter by some constant, irrespective of the scale parameter's value. For instance, changing a time constant from 2 seconds to 2.2 seconds can be expected to have about the same qualitative effect as changing it from 200 to 220 . Other properties of scale parameters is that they must not become negative, and that as they approach zero, the dynamics of the associated system becomes more and more sensitive to small changes. This means that in practive one must add machinery to enforce the constraint of positiveness, and that gradient descent will become increasingly unstable as a scale parameter approaches zero, due to the system's growing sensitivity to its value. Also, the flat prior is no longer the appropriate zero-knowledge prior.

All these problems can be solved in a single stroke by noting that the correct zero-knowledge hypothesis for scale parameters is not flat in their values, but rather flat in their log values [131]. In practice, This corresponds to doing gradient descent in $\mathcal{L}_{T}=\log T$ rather than in $T$ itself; in other words, to not manipulating $T$ directly but rather using $\Delta \mathcal{L}_{T}=-\eta d E / d \mathcal{L}_{T}$. Such a policy also solves the practical problems with scale parameters noted above, as it makes the gradient descent process stiffer as $T$ approaches zero, compensating for the system's increased sensitivity in that region, and it naturally enforces $T>0$ since $T=\exp \mathcal{L}_{T}>0$, which enforces this constraint without any additional mechanism. This last property led to the independent invention and use of this technique by [132].

In addition, weight decay of scale parameters becomes simpler, as decaying $\mathcal{L}_{T}$ towards zero corresponds to decaying $T$ towards one, which is a reasonable target. Of course, a constant factor can be inserted to make the decay towards some other a priori most likely value. Note, however, that the force exerted by the decay term will scale with the log parameter, which is more appropriate, since the additional force exerted should correspond to the change's effect on the dynamics of the system, in order to pass dimensional analysis.

\section{Summary and Conclusion}

\section{A. Complexity Comparison}

Consider a network with $n$ units and $m$ weights which is run for $s$ time steps (variable grid methods [133] would re- 
duce $s$ by dynamically varying $\Delta t$ ) where $s=\left(t_{1}-t_{0}\right) / \Delta t$. Additionally, assume that the computation of each $e_{i}(t)$ is $O(1)$ and that the network is not partitioned.

Under these conditions, simulating the $\mathbf{y}$ system takes $O(m+n)=O(m)$ time for each time step, as does simulating the $\mathbf{z}$ system. This means that using the technique described in section IV, the entire simulation takes $O(m)$ time per time step, the best that could be hoped for. Storing the activations and weights takes $O(n+m)=O(m)$ space, and storing $\mathbf{y}$ during the forward simulation to replay while simulating $\mathbf{z}$ backwards takes $O(s n)$ space, so if we use this technique the entire computation takes $O(s n+m)$ space. If we simulate $\mathbf{y}$ backwards during the backwards simulation of $\mathbf{z}$, the simulation requires $O(n+m)$ space, again the best that could be hoped for. This later technique, however, is susceptible to numeric stability problems.

The online technique of RTRL described in section III-B requires $O\left(n^{2} m\right)$ time each time step, and $O(\mathrm{~nm})$ space. The other techniques discussed in that section require less time and space, and retain all of the advantages of being online (with the possible exception of simplicity of implementation), so it would appear that these new online methods dominate RTRL. These time complexity results are for sequential machines, and are summarized in table I.

Note that in this section we are concerning ourselves with how much computation it takes to obtain the gradient information. This is generally just the inner loop of a more complex algorithm to adjust the weights, which uses the gradient information, such as a stochatic gradient descent algorithm.

\section{B. Speeding the Optimization}

Experience has shown that learning in these networks tends to be "stiff" in the sense that the Hessian of the error with respect to the weights (the matrix of second derivatives) tends to have a wide eigenvalue spread. One technique that has proven useful in this particular situation is that of [134] which was applied by Fang and Sejnowski to the single figure eight problem perturbed in figure 9 with great success by [135]. For a modern variant of this technique which is suitable to online pattern presentation, see [136], [137], [138].

Since the acceleration of convergence in these gradient systems is such an important issue, it can be helpful to know some of the techniques used to analyze the limitations of convergence under various conditions in systems of this sort, and of some other techniques for accelerating their convergence; see [139, page 304] and [140], [141], [142], [143], [144], [145], [146], [147], [148].

\section{Prospects and Future Work}

Control domains are the most natural application for continous time recurrent networks, but signal processing and speech generation (and recognition using generative techniques) are also domains to which this type of network might be naturally applied. Such domains may lead us to complex architectures like those discussed in section III-E. For control domains, it seems important to have ways to force the learning towards solutions that are stable in the control sense of the term.

On the other hand, we can turn the logic of section $\mathrm{V}$ around. Consider a difficult constraint satisfaction task of the sort that neural networks are sometimes applied to, such as the traveling salesman problem [149]. Two competing techniques for such problems are simulated annealing [150], [58] and mean field theory [92]. By providing a network with a noise source which can be modulated (by second order connections, say) we could see if the learning algorithm constructs a network that makes use of the noise to generate networks that do simulated annealing, or if pure gradient descent techniques are evolved. If a hybrid network evolves, its structure may give us insight into the relative advantages of these two different optimization techniques, and into the best ways to structure annealing schedules.

\section{Conclusions}

Recurrent networks are often avoided because of a fear of inordinate learning times and incomprehensible algorithms and mathematics. It should be clear from the above that such fears are unjustified. Certainly there is no reason to use a recurrent network when a feedforward layered architecture suffices; but on the other hand, if recurrence is needed, there are a plethora of learning algorithms available across the spectrum of quiescence vs. dynamics and across the spectrum of accuracy vs. complexity and across the spectrum of space vs. time. These new learning algorithms, and experience with recurrent and temporally continuous networks, has made them much more tractable and practical than they seemed only a few years ago.

\section{Acknowledgments}

For provocative and enlightening discussions, I would like to thank Lee Giles, Geoff Hinton, Gary Kuhn, Fernando Pineda, Richard Szeliski, David Touretzky, Ron Williams, and members of the old Boltzmann group at CMU. I would also like to thank a number of anonymous reviewers. This research was sponsered in part by NSF EET-8716324, by ONR N00014-86-K-0678, and by a Fannie and John Hertz Foundation Fellowship.

\section{REFERENCES}

[1] J. L. McClelland, D. E. Rumelhart, and G. E. Hinton, "The appeal of parallel distributed processing", In Rumelhart et al. [151].

[2] D. Marr and T. Poggio, "Cooperative computation of stereo disparity", Science, vol. 194, pp. 283-287, 1976.

[3] G. Marr, D. Palm and T. Poggio, "Analysis of a cooperative stereo algorithm", Biological Cybernetics, vol. 28, pp. 223-229, 1978.

[4] Geoffrey E. Hinton, Relaxation and its role in vision, $\mathrm{PhD}$ thesis, University of Edinburgh, 1977, Described in [152, pages 408-430].

[5] L. S. Davis and A. Rosenfeld, "Cooperating processes for lowlevel vision: A survey", Artificial Intelligence, vol. 3, pp. 245264, 1981.

[6] Richard Szeliski, "Cooperative algorithms for solving randomdot stereograms", Tech. Rep. CMU-CS-86-133, Carnegie Mellon University, 1986. 


\begin{tabular}{|l||c|c|c|c|c|c|}
\hline technique & time/ $/ \mathrm{t}$ & space & online & stable & local & exact \\
\hline \hline BPTT, storing $y$ & $O(m)$ & $O(s n+m)$ & no & yes & yes & yes \\
\hline RTRL & $O\left(n^{2} m\right)$ & $O(n m)$ & yes & yes & no & yes \\
\hline BPTT, only $h$ steps & $O(h m)$ & $O(h n+m)$ & yes & yes & yes & no \\
\hline Williams-Peng, $h$ steps & $O(m)$ & $O(h n+m)$ & yes & yes & yes & no \\
\hline hybrid BPTT/RTRL & $O(n m)$ & $O(n m)$ & yes & yes & no & yes \\
\hline Sun-Chen-Lee & $O(n m)$ & $O\left(n^{2}+m\right)$ & yes & yes & no & yes \\
\hline BPTT, recalc. $y$ & $O(m)$ & $O(m)$ & no & no & yes & yes \\
\hline
\end{tabular}

TABLE I

A SUMMARY OF THE COMPLEXITy OF SOME LEARNING PROCEDURES FOR RECURRENT NETWORKS. IN THE "STORING $y$ " TECHNIQUE WE STORE $y$ AS TIME IS RUN FORWARDS AND REPLAY IT AS WE RUN TIME BACKWARDS COMPUTING $z$. IN “ $y$ BACKWARDS" WE DO NOT STORE $y$, INSTEAD RECOMPUTING IT AS TIME IS RUN BACKWARDS. "FORWARD PROFAGATION" 1 AND 2 ARE THE ONLINE TECHNIQUES DESCRIBED IN SECTION III-B. THE TIMES GIVEN ARE FOR COMPUTING THE GRADIENT WiTH RESPECT TO ONE PATTERN.

[7] Geoffrey E. Hinton, "Using relaxation to find a puppet", in Proceedings of the A.I.S.B. Summer Conference, University of Edinburgh, July 1976.

[8] D. L. Waltz and J. B. Pollack, "Massively parallel parsing: A strongly interactive model of natural language interpretation", Cognitive Science, vol. 9, pp. 51-74, 1985.

[9] Ning Qian and Terrence J. Sejnowski, "Learning to solve random-dot stereograms of dense and transparent surfaces with recurrent backpropagation", in Proceedings of the 1988 Connectionist Models Summer School, David S. Touretzky, Geoffrey E. Hinton, and Terrence J. Sejnowski, Eds. 1989, pp. 435443, Morgan Kaufmann.

[10] O. Nerrand, P. Roussel-Ragot, G. Dreyfus L. Personnaz, and S. Marcos, "Neural networks and non-linear adaptive filtering: Unifying concepts and new algorithms", Neural Computation, vol. 5, no. 2, pp. 165-197, 1993.

[11] T. W. Karjala, D. M. Himmelblau, and R. Miikkulainen, "Data rectification using recurrent (Elman) neural networks", In IJCNN92'Baltimore [153], pp. 901-905.

[12] Raymond L. Watrous, Bruce Laedendorf, and Gary M. Kuhn, "Complete gradient optimization of a recurrent network applied to BDG descrimination", Journal of the Acoustical Society of America, vol. 87, no. 3, pp. 1301-1309, Mar. 1990.

[13] P. Poddar and K.P. Unnikrishnan, "Nonlinear prediction of speech signals using memory neuron networks", In Juang et al. [31], pp. 395-404.

[14] Dario Albesano adn Roberto Gemello and Franco Mana, "Word recognition with recurrent network automata", In IJCNN92'Baltimore [153], pp. 308-313.

[15] Shawn R. Lockery, Yan Fang, and Terrence J. Sejnowski, "A dynamic neural network model of sensorimotor transformations in the leech", Neural Computation, vol. 2, no. 3, pp. 274-282, 1990.

[16] Kenji Doya, M. E. T. Boyle, and A. I. Selverston, "Mapping between neural and physical activities of the lobster gastric mill system", In Hanson et al. [154], pp. 913-920.

[17] Kenji Doya, A. I. Selverston, and P. F. Rowat, "A hodgkinhuxley type neuron model that learns slow non-spike oscillation", In Cowan et al. [155].

[18] Yves

Chauvin and David E. Rumelhart, Eds., Back-propagation: Theory, Architectures and Applications, Lawrence Erlbaum Associates, 1995.

[19] Paul R. Gorman and Terrence J. Sejnowski, "Analysis of hidden units in a layered network trained to classify sonar targets", Neural Networks, vol. 1, no. 1, pp. 75-89, 1988.

[20] Kevin Lang and Geoffrey Hinton, "The development of the time-delay neural network architecture for speech recognition", Tech. Rep. CMU-CS-88-152, Department of Computer Science, Carnegie Mellon University, Nov. 1988.

[21] Alex Waibel, T. Hanazawa, G. Hinton, K. Shikano, and K. Lang, "Phoneme recognition using time-delay networks", IEEE Transactions on Acoustics, Speech, and Signal Processing, vol. 37, no. 3, pp. 328-339, 1989.
[22] Kevin J. Lang, Geoffrey E. Hinton, and Alex Waibel, "A timedelay neural network architecture for isolated word recognition", Neural Networks, vol. 3, no. 1, pp. 23-43, 1990.

[23] Kevin J. Lang and Geoffrey E. Hinton, "Dimensionality reduction and prior knowledge in e-set recognition", In Touretzky [156], pp. $178-185$.

[24] D. W. Tank and J. J. Hopfield, "Concentrating information in time: Analog neural networks with applications to speech recognition problems", In Caudill and Butler [157], pp. 455468.

[25] Shawn P. Day and Michael R. Davenport, "Continuoustime temporal backpropagation with adaptable time delays", IEEE Transactions on Neural Networks, , no. 2, pp. 348-354, 1993, Ftp archive.cis.ohio-state.edu: /pub/neuroprose/day.temporal.ps.Z.

[26] M. I. Jordan, "Generic constraints on underspecified target trajectories", In IJCNN89 [158].

[27] Applications of Artificial Neural Networks, number 1294 in APIE Proceedings Series, Orlando, Florida, Apr. 18-20 1990.

[28] A. S. Weigend, D. E. Rumelhart, and B. A. Huberman, "Generalization by weight-elimination with application to forecasting", In Lippmann et al. [159], pp. 875-882.

[29] Bert de Vries and Jose C. Principe, "A theory for neural networks with time delays", In Lippmann et al. [159], pp. 162-168.

[30] A.D. Back and A.C. Tsoi, "FIR and IIR synapses, a new neural network architecture for time series modelling", Neural Computation, vol. 3, no. 3, pp. 337-350, 1991.

[31] B.H. Juang, S.Y. Kung, and C A. Camm, Eds., Neural Networks for Signal Processing: Proceedings of the 1991 IEEE Workshop. IEEE Press, 1991.

[32] D. Hush and B. Horne, "Progress in supervised neural networks", IEEE Signal Processing Magazine, vol. 10, no. 1, pp. $8-39,1993$.

[33] B. de Vries and J. Principe, "The gamma model-a new neural network for temporal processing", Neural Networks, vol. 5, no. 4, pp. 565-576, 1992.

[34] T. Maxwell, C. L. Giles, Y. C. Lee, and H. H. Chen, "Nonlinear dynamics of artificial neural systems", In Denker [160], pp. 299-304.

[35] Y. LeCun, B. Boser, J. S. Denker, D. Henderson, R. E. Howard, W. Hubbard, and L. D. Jackel, "Backpropagation applied to handwritten zip code recognition", Neural Computation, vol. 1, no. 4, pp. 541-551, 1989.

[36] M. Kawato, T. Setoyama, and R. Suzuki, "Feedback error learning of movement by multi-layer neural networks", in Proceedings of the International Neural Networks Society First Annual Meeting, 1988, p. 342

[37] M. I. Jordan and R. A. Jacobs, "Learning to control an unstable system with forward modeling", In Touretzky [156].

[38] K.S. Narendra and K. Parthasarathy, "Identification and control of dynamical systems using neural networks", IEEE Transactions on Neural Networks, vol. 1, pp. 4-27, Mar. 1990.

[39] W. Thomas Miller, III, Richard S. Sutton, and Paul J. Werbos, Eds., Neural Networks for Control, MIT Press, 1990. 
[40] H. T. Siegelmann and E. D. Sontag, "Turing computability with neural networks", Applied Mathematics Letters, vol. 4, no. 6 , pp. $77-80,1991$.

[41] J. Kilian and H. T. Siegelmann, "Computability with the classical sigmoid", in Sixth Annual ACM Workshop on Computational Learning Theory, Santa Cruz, CA, July 1993, pp. 137143.

[42] H. T. Siegelmann and E. D. Sontag, "Analog computation via neural networks", in The Second Israel Symposium on Theory of Computing and Systems, Natanya, Israel, June 1993, To appear in Theoretical Computer Science.

[43] Axel Cleeremans, David Servan-Schreiber, and James McClelland, "Finite state automata and simple recurrent networks", Neural Computation, vol. 1, no. 3, pp. 372-381, 1989.

[44] Raymond L. Watrous and G. M. Kuhn, "Induction of finitestate automata using second-order recurrent networks", In Moody et al. [161], pp. 309-316.

[45] C. L. Giles, C. B. Miller, D. Chen, G. Z. Sun, H. H. Chen, and Y. C. Lee, "Extracting and learning an unknown grammar with recurrent neural networks", In Moody et al. [161], pp. 317-324.

[46] Michael C. Mozer and Sreerupa Das, "A connectionist symbol manipulator that discovers the structure of context-free languages", In Hanson et al. [154], pp. 863-870.

[47] Sreerupa Das, C. Lee Giles, and Guo-Zheng Sun, "Using prior knowledge in a NNPDA to learn context-free languages", In Hanson et al. [154], pp. 65-72.

[48] John F. Kolen, "Fool's gold: Extracting finite state machines from recurrent network dynamics", In NIPS*93 [155], Ftp archive.cis.ohio-state.edu: /pub/neuroprose/kolen.foolsgold.ps.Z.

[49] Sreerupa Das and Michael C. Mozer, "A unified gradientdescent/clustering architecture for finite state machine induction", In Cowan et al. [155].

[50] Dana Angluin, "Learning regular sets from queries and counterexamples", Information and Computation, vol. 75, pp. 87$106,1987$.

[51] Kevin J. Lang, "Random dfa's can be approximately learned from sparse uniform examples", in Fifth Annual ACM Workshop on Computational Learning Theory, Pittsburgh, PA, July 1992, pp. 45-52.

[52] Josef Hochreiter, "Untersuchungen zu dynamischen neuronalen netzen", 1991, Diplomarbeit, Institut für Informatik, Lehrstuhl Prof. Brauer, Technische Universität München.

[53] Michael C. Mozer, "Induction of multiscale temporal structure", In Moody et al. [161], pp. 275-282.

[54] Jürgen H. Schmidhuber, "Learning complex, extended sequences using the principle of history compression", Neural Computation, vol. 4, no. 2, pp. 234-242, 1992.

[55] Jürgen H. Schmidhuber, "Learning unambiguous reduced sequence descriptions", In Moody et al. [161], pp. 291-298.

[56] Geoffrey E. Hinton, "Learning distributed representations of concepts", in Proceedings of the Eighth Annual Cognitive Science Conference. 1986, Lawrence Erlbaum Associates.

[57] T.J. Sejnowski, P.K. Kienker, and G. Hinton, "Learning symmetry groups with hidden units: Beyond the perceptron", Physica D, vol. 22, pp. 260-275, 1986.

[58] David H. Ackley, Geoffrey E. Hinton, and Terrence J. Sejnowski, "A learning algorithm for Boltzmann Machines", Cognitive Science, vol. 9, pp. 147-169, 1985 .

[59] David E. Rumelhart, Geoffrey E. Hinton, and R. J. Williams, "Learning internal representations by error propagation", In Rumelhart et al. [151].

[60] Paul J. Werbos, Beyond Regression: New Tools for Prediction and Analysis in the Behavioral Sciences, PhD thesis, Harvard University, 1974.

[61] David B. Parker, "Learning-logic", Tech. Rep. TR-47, MIT Center for Research in Computational Economics and Management Science, 1985.

[62] R. Howard, Dynamic Programming and Karkhov Processes, MIT Press, 1960.

[63] D.S. Touretzky and D.A. Pomerleau, "What's hidden in the hidden layers?", BYTE, pp. 227-233, Aug. 1989.

[64] B. Widrow and M. Hoff, "Adaptive switching circuits", in Western Electronic Show and Convention, Convention Record. 1960, vol. 4, pp. 96-104, Institute of Radio Engineers (now IEEE).
[65] Yann LeCun, Ido Kanter, and Sara A. Solla, "Second order properties of error surfaces: Learning time and generalization", In Lippmann et al. [159], pp. 918-924.

[66] B. Baird, "A learning rule for CAM storage of continuous periodic sequences", In IJCNN90 II [162], pp. 493-498.

[67] Bill Baird and Frank Eeckman, "CAM storage of analog patterns and continuous sequences with $3 n^{2}$ weights", In Lippmann et al. [159], pp. 91-97.

[68] C. A. Skarda and W. J. Freeman, "How brains make chaos in order to make sense of the world", Brain and Behavioral Science, vol. 10, Nov. 1987.

[69] W. J. Freeman, "Simulation of chaotic EEG patterns with a dynamic model of the olfactory system", Biological Cybernetics, vol. 56, pp. 139, 1987.

[70] J. P. Crutchfield and B. S. McNamara, "Equations of motion from a data series", Complex Systems, vol. 1, pp. 417-452, 1987.

[71] Alan Lapedes and Robert Farber, "Nonlinear signal processing using neural networks: Prediction and system modelling", Tech. Rep. LA-UR-87-2662, Theoretical Division, Los Alamos National Laboratory, Los Alamos, NM, 1987.

[72] Fernando Pineda, "Generalization of back-propagation to recurrent neural networks", Physical Review Letters, vol. 19, no. 59, pp. 2229-2232, 1987

[73] L. B. Almeida, "A learning rule for asynchronous perceptrons with feedback in a combinatorial environment", In Caudill and Butler [157], pp. 609-618.

[74] Geoffrey E. Hinton, "Deterministic Boltzmann learning performs steepest descent in weight-space", Neural Computation, vol. 1, no. 1, pp. 143-150, 1989.

[75] Pierre Baldi and Fernando Pineda, "Contrastive learning and neural oscillations", Neural Computation, vol. 3, no. 4, pp. 526-545, 1991.

[76] Barak A. Pearlmutter, "Dynamic recurrent neural networks", Tech. Rep. CMU-CS-90-196, Carnegie Mellon University School of Computer Science, Pittsburgh, PA, Dec. 1990.

[77] Marco Gori, Yoshua Bengio, and Renato de Mori, "BPS: A learning algorithm for capturing the dynamic nature of speech", In IJCNN89 [158], pp. 417-423.

[78] Gary Kuhn, "A first look at phonetic discrimination using connectionist models with recurrent links", SCIMP working paper 82018, Institute for Defense Analysis, Princeton, New Jersey, Apr. 1987.

[79] Michael C. Mozer, "A focused backpropagation algorithm for temporal pattern recognition", Complex Systems, vol. 3, no. 4, pp. 349-381, Aug. 1989.

[80] Tadasu Uchiyama, Katsunori Shimohara, and Yukio Tokunaga, "A modified leaky integrator network for temporal pattern recognition", In IJCNN89 [158], pp. 469-475.

[81] Barak A. Pearlmutter, "Two new learning procedures for recurrent networks", Neural Network Review, vol. 3, no. 3, pp. 99-101, 1990.

[82] William H. Press, Brian P. Flannery, Saul A. Teukolsky, and William T. Verrerling, Numerical Recipes in C, Cambridge University Press, 1988.

[83] M. A. Cohen and Steven Grossberg, "Stability of global pattern formation and parallel memory storage by competitive neural networks", IEEE Transactions on Systems, Man and Cybernetics, vol. 13, pp. 815-826, 1983.

[84] Geoffrey E. Hinton and Terrence J. Sejnowski, "Optimal perceptual inference", in Proceedings of the IEEE conference on Computer Vision and Pattern Recognition, Washington DC, June 1983, IEEE Computer Society, pp. 448-453.

[85] Amir F. Atiya, "Learning on a general network", In Anderson [163], pp. 22-30.

[86] Steve Renals and Richard Rohwer, "A study of network dynamics", Journal of Statistical Physics, vol. 58, pp. 825-848, June 1990.

[87] Robert B. Allen and Joshua Alspector, "Learning of stable states in stochastic asymmetric networks". Tech. Rep. TMARH-015240, Bell Communications Research, Morristown, NJ, Nov. 1989

[88] Conrad C. Galland and Geoffrey E. Hinton, "Deterministic Boltzmann learning in networks with asymmetric connectivity", Tech. Rep. CRG-TR-89-6, University of Toronto Department of Computer Science, 1989 
[89] Steven J. Nowlan, "Gain variation in recurrent error propagation networks", Complex Systems, vol. 2, no. 3, pp. 305-320, June 1988 .

[90] Mary B. Ottaway, Patrice Y. Simard, and Dana H. Ballard, "Fixed point analysis for recurrent neural networks", in $A d$ vances in Neural Information Processing Systems I, David S. Touretzky, Ed. 1989, Morgan Kaufmann.

[91] C. Peterson and J. R. Anderson, "A mean field theory learning algorithm for neural nets", Complex Systems, vol. 1, 1987.

[92] C. Peterson and James R. Anderson, "A mean field theory learning algorithm for neural networks", Tech. Rep. EI-259-87, MCC, Aug. 1987.

[93] Geoffrey E. Hinton and Kevin J. Lang, "Shape recognition and illusory conjunctions", in the Ninth International Joint Conference on Artificial Intelligence, Los Angeles, Aug. 1985, vol. 1, pp. 252-259, Morgan Kaufmann.

[94] T. J. Sejnowski, "Higher-order Boltzmann machines", In Denker [160], pp. 398-403.

[95] Paul J. Werbos, "Backpropagation through time: what it does and how to do it", Proceedings of the IEEE, vol. 78, pp. 15501560, 1990.

[96] Barak Pearlmutter, "Learning state space trajectories in recurrent neural networks", Neural Computation, vol. 1, no. 2, pp. 263-269, 1989

[97] Paul J. Werbos, "Generalization of backpropagation with application to a recurrent gas market model", Neural Networks, vol. 1, pp. 339-356, 1988.

[98] Arthur E. Bryson, Jr., "A steepest ascent method for solving optimum programming problems", Journal of Applied Mechanics, vol. 29, no. 2, pp. 247, 1962 .

[99] Stuart E. Dreyfus, Dynamic Programming and the Calculus of Variations, vol. 21 of Mathematics in science and engineering. Academic Press, 1965.

[100] Paul J. Werbos, "Applications of advances in nonlinear sensitivity analysis", in System Modeling and Optimization: Proceedings of the 10th IFIP Conference, R. F. Drenick and F. Kozin, Eds., (New York, Aug 31-Sep 4, 1981), 1982, number 38 in Lecture Notes in Control and Information Sciences, SpringerVerlag.

[101] A. J. Robinson and F. Fallside, "Static and dynamic error propagation networks with application to speech coding", In Anderson [163], pp. 632-641.

[102] Michael Gherrity, "A learning algorithm for analog, fully recurrent neural networks", In IJCNN89 [158], pp. 643-644.

[103] Ronald J. Williams and David Zipser, "A learning algorithm for continually rumning fully recurrent neural networks", Neural Computation, vol. 1, no. 2, pp. 270-280, 1989.

[104] K. S. Narendra and K. Parthasarathy, "Gradient methods for the optimization of dynamical systems containing neural networks", IEEE Transactions on Neural Networks, vol. 2, no. 2, pp. 252-262, Mar. 1991.

[105] D. H. Jacobson, "New second order and first order algorithm for determining optimal control: A differential dynamic programming approach", Journal of Optimization Theory and Applications, vol. 2, 1968.

[106] R. E. Bellman, Methods of Nonlinear Aralysis: Volume II Academic Press, 1973.

[107] A. Gelb et al., Eds., Applied Optimal Estimation, MIT Press, 1974.

[108] David Zipser, "Subgrouping reduces complexity and speeds up learning in recurrent networks", In Touretzky [156], pp. 638641.

[109] Ronald J. Williams and Jing Peng, "An efficient gradient-based algorithm for on-line training of recurrent network trajectories", Neural Computation, vol. 2, no. 4, pp. 490-501, 1990.

[110] R. J. Williams and D. Zipser, "Gradient-based learning algorithms for recurrent networks and their computational complexity", In Chauvin and Rumelhart [18], Also published as [164].

[111] Jürgen H. Schmidhuber, "A fixed size storage $O\left(n^{3}\right)$ time complexity learning algorithm for fully recurrent continually running networks", Neural Computation, vol. 4, no. 2, pp. 243-248, 1992.

[112] Guo-Zheng Sun, Hsing-Hen Chen, and Yee-Chun Lee, "Green's function method for fast on-line learning algorithm of recurrent neural networks", In Moody et al. [161], pp. 333-340.
[113] U. Bodenhausen, "Learning internal representations of pattern sequences in a neural network with adaptive time-delays", In IJCNN90 II [162].

[114] D. W. Tank and J. J. Hopfield, "Neural computation by time compression", Proceedings of the National Academy of Sciences, vol. 84, pp. 1896-1900, 1987.

[115] R. L. Watrous, Speech Recognition Using Connectionist Networks, PhD thesis, University of Pennsylvania, Oct. 1988.

[116] B. J. Norris, M. J. Coleman, and M. P. Nusbaum, "Distinct responses of electrically-coupled pacemaker neurons to activation of a modulatory projection neuron", Society for Neuroscience Abstracts, vol. 20, no. 18.6, pp. 23, 1994.

[117] Patrice Y. Simard, Jean Pierre Rayzs, and Bernard Victorri, "Shaping the state space landscape in recurrent networks", In Lippmann et al. [159], pp. 105-112.

[118] J.L. Elman, "Finding structure in time", Cognitive Science, vol. 14, pp. 179-211, 1990.

[119] Jeffrey L. Elman, "Finding structure in time", Tech. Rep. CRL8801, Center for Research in Language, UCSD, Apr. 1988.

[120] Yann LeCun, "Une procédure d'apprentissage pour réseau à seuil assymétrique", in Cognitiva 85: A la Frontiére de l'Intelligence Artificielle des Sciences de la Connaissance des Neurosciences, Paris 1985, 1985, pp. 599-604, CESTA, Paris.

[121] T. Grossman, R. Meir, and E. Domany, "Learning by choice of internal representations", Complex Systems, vol. 2, pp. 555$575,1989$.

[122] Richard Rohwer, "The "moving targets" training algorithm", In Touretzky [156], pp. 558-565.

[123] Ronald J. Williams and David Zipser, "A learning algorithm for continually running fully recurrent neural networks", Tech. Rep. ICS Report 8805, UCSD, La Jolla, CA 92093, Nov. 1988.

[124] Michael I. Jordan, "Attractor dynamics and parallelism in a connectionist sequential machine", in Proceedings of Ninth $A n-$ nual Conference of the Cognitive Science Society. 1986, pp. 531-546, Lawrence Erlbaum Associates.

[125] M. B. Matthews, "Neural network nonlinear adaptive filtering using the extended Kalman filter algorithm", in Proceedings of the International Neural Networks Conference, Paris, France, July 1990, vol. 1, pp. 115-119.

[126] Ronald J. Williams, "Training recurrent networks using the extended Kalman filter", In IJCNN92'Baltimore [153], pp. 241250 .

[127] R. E. Kalman. "A new approach to linear filtering and prediction problems", Trans. ASME Journal of Basic Engineering, vol. 82 , no. 1, pp. 35-45, Mar. 1960.

[128] R. K. Mahra, "On the identification of variances and adaptive Kalman filtering", IEEE Transactions on Automatic Control, vol. AC-15, no. 2, pp. 175-184, Apr. 1970.

[129] B. D. O. Anderson and J. B. Moore, Optimal Filtering, Prentice-Hall, 1979.

[130] S. J. Nowlan and G. E. Hinton, "Adaptive soft weight tying using gaussian mixtures", In Moody et al. [161], pp. 993-1000.

[131] John Skilling, Ed., Maximum Entropy and Bayesian Methods. Kluwer Academic Publishers, 1989.

[132] P. F. Rowat and A. I. Selverston, "Learning algorithms for oscillat ory networks with gap junctions and membrane currents", Network: Computation in Neural Systems, vol. 2, no. 1, pp. 17-42, Feb. 1991.

[133] J. G. Blom, J. M. Sanz-Serna, and Jan G. Verwer, On Simple Moving Grid Methods for One-Dimensional Evolutionary Partial Differential Equations, Stichting Mathematisch Centrum, Amsterdam. The Netherlands, 1986.

[134] Robert A. Jacobs, "Increased rates of convergence through learning rate adaptation", Neural Networks, vol. 1, no. 4, pp. 295-307, 1988

[135] Yan Fang and Terrence J. Sejnowski, "Faster learning for dynamic recurrent backpropagation", Neural Computation, vol. 2, no. 3, pp. 270-273, 1990.

[136] Richard S. Sutton, "Gain adaptation beats least squares?", in Seventh Yale Workshop on Adaptive and Learning Systems, 1992, pp. 161-166.

[137] Richard S. Sutton, "Adapting bias by gradient descent: An incremental version of delta-bar-delta", in Proceedings of the National Conference on Artificial Intelligence AAAI-92, 1992.

[138] Mark A. Gluck, Paul T. Glauthier, and Richard S. Sutton, "Adaptation of cue-specific learning rates in network models of human category learning", in Proceedings of the Fourteenth 
Annual Meeting of the Cognitive Science Society, Bloomington, IN, 1992.

[139] Douglass J. Wilde and Charles S. Beightler, Foundations of Optimization, Prentice-Hall, 1967.

[140] B. Widrow, J. M. McCool, M. G. Larimore, and C. R. Johnson, Jr., "Stationary and nonstationary learning characteristics of the LMS adaptive filter", Proceedings of the IEEE, vol. 64, pp. 1151-1162, 1976

[141] Bernard Widrow and Samuel D. Stearns, Adaptive Signal Processing, Prentice-Hall signal processing series. Prentice-Hall, 1985.

[142] S. Thomas Alexander, Adaptive Signal Processing, SpringerVerlag, 1986.

[143] David B. Parker, "Optimal algorithms for adaptive networks: Second order back propagation, second order direct propagation, and second order Hebbian learning", In Caudill and Butler [157], pp. 593-600.

[144] Raymond Watrous, "Learning algorithms for connectionist networks: Applied gradient methods of nonlinear optimization", In Caudill and Butler [157], pp. 619-627.

[145] J. J. Shynk and S. Roy, "The LMS algorithm with momentum updating", in Proceedings of the IEEE International Symposium on Circuits and Systems, June 6-9 1988, pp. 2651-2654.

[146] Gerald Tesauro, Yu He, and Subutai Ahmad, "Asymptotic convergence of backpropagation", Neural Computation, vol. 1 . no. 3, pp. 382-391, 1989.

[147] Mehmet Ali Tuğay and Yalçin Tanik, "Properties of the momentum LMS algorithm", Signal Processing, vol. 18, no. 2, pp. $117-127$, Oct. 1989.

[148] Barak A. Pearlmutter, "Gradient descent: Second-order momentum and saturating error", In Moody et al. [161], pp. 887894.

[149] J. J. Hopfield and D. W. Tank, “"Neural' computation of decisions in optimization problems", Biological Cybernetics, vol. 52 , pp. $141-152,1985$.

[150] S. Kirkpatrick, C. D. Gelatt, Jr., and M. P. Vecchi, "Optimization by simulated annealing", Science, vol. 220, pp. 671-680, 1983.

[151] D. E. Rumelhart, J. L. McClelland, and the PDP research group., Eds., Parallel distributed processing: Explorations in the microstructure of cognition, Volume 1: Foundations, MIT Press, 1986.

[152] D. H. Ballard and C. M. Brown, Computer Vision, PrinticeHall, 1982.

[153] International Joint Conference on Neural Networks, Baltimore, MD, Apr. 1992. IEEE Press.

[154] Stephen José Hanson, Jack D. Cowan, and C. Lee Giles, Eds. Advances in Neural Information Processing Systems 5. Morgan Kaufmann, 1993

[155] Jack D. Cowan, Gerald Tesauro, and Joshua Alspector, Eds., Advances in Neural Information Processing Systems 6. Morgan Kaufmann, 1994

[156] David S. Touretzky, Ed., Advances in Neural Information Processing Systems 2. Morgan Kaufmann, 1990.

[157] Maureen Caudill and Charles Butler, Eds., IEEE First International Conference on Neural Networks, San Diego, CA, June 21-24 1987.

[158] International Joint Conference on Neural Networks, Washington DC, June 18-22 1989. IEEE Press.

[159] Richard P. Lippmann, John E. Moody, and David S. Touretzky, Eds., Advances in Neural Information Processing Systems 3. Morgan Kaufmann, 1991.

[160] John S. Denker, Ed., Snowbird Conference on Neural Networks for Computing, number 151 in AIP conference proceedings. American Institute of Physics, 1986.

[161] John E. Moody, Steven J. Hanson, and Richard P. Lippmann, Eds., Advances in Neural Information Processing Systems 4. Morgan Kaufmann, 1992.

[162] International Joint Conference on Neural Networks, San Diego, CA, June 1990. IEEE Press.

[163] Dana Z. Anderson, Ed., Neural Information Processing Systems, New York, New York, 1988. American Institute of Physics.

[164] R. J. Williams and D. Zipser, "Gradient-based learning algorithms for recurrent connectionist networks", Tech. Rep. NUCCS-90-9, College of Computer Science, Northeastern University, Boston, MA, 1990, Also published as [110].
Barak A. Pearlmutter has been a connectionist for over a decade, and will receive his $\mathrm{PhD}$ in Computer Science from Carnegie Mellon University really really soon (really mom, I promise.) When paging through IEEE transactions, he reads the bio's first. He can slice a hippocampus, train an artificial neural network, implement Scheme, and get a giga-ohm seal on a cultured frog neuron with a single suck. 